\title{
A sequential formula for electronic coupling in long range bridge-assisted electron transfer: Formulation of theory and application to alkanethiol monolayers
}

\author{
Chao-Ping Hsu and R. A. Marcus \\ Arthur Amos National Laboratory of Chemical Physics, 127-72, California Institute of Technology, \\ Pasadena, California 91125
}

(Received 23 May 1996; accepted 3 October 1996)

\begin{abstract}
A recursion relation is formulated for the Green's function for calculating the effective electron coupling in bridge-assisted electronic transfer systems, within the framework of the tight-binding Hamiltonian. The recursion expression relates the Green's function of a chain bridge to that of the bridge that is one unit less. It is applicable regardless of the number of orbitals per unit. This method is applied to the system of a ferrocenylcarboxy-terminated alkanethiol on the Au(111) surface. At larger numbers of bridge units, the effective coupling strength shows an exponential decay as the number of methylene $\left(-\mathrm{CH}_{2}-\right)$ units increases. This sequential formalism shows numerical stability even for a very long chain bridge and, since it uses only small matrices, requires much less computer time for the calculation. Identical bridge units are not a requirement, and so the method can be applied to more complicated systems. (C) 1997 American Institute of Physics.
\end{abstract} [S0021-9606(97)01802-3]

\section{INTRODUCTION}

Electron transfer(ET) over long distances has been studied extensively in recent experimental and theoretical works, both in homogeneous systems ${ }^{1-5}$ and across monolayers on electrodes ${ }^{6-9}$ Some of the work addressed the importance of the role played by long range ET reactions in biological processes while others demonstrated the underlying fundamental properties of such reactions. Many of these studies suggest that typically the rate has exponential dependence on the distance between donor $D$ and acceptor $A$. Theoretical studies $^{2,10}$ on molecular wires with one orbital representing each site of the wires, show exponential dependence of the conductance with the length of wire when the electron is at an energy outside of the wire's energy band, and large conductance is obtained with oscillatory dependence on wire length for the energy of an electron inside the wire's band. A sequential treatment is formulated here for electron transfer through a linear chain bridge that is allowed to have more than one orbital in each site. When the energy of $D$ and $A$ states lies out of the "energy band" of a long chain bridge, the well known exponential dependence of the matrix element on distance is expected.

For the coupling of the electronic and nuclear motion a Golden rule treatment has given a satisfactory description of the non-adiabatic reaction rate for weak (i.e., long range) coupling. In this case, the rate constant $k$ for electron transfer from an electronic state of the donor to a state of the acceptor is given by:

$$
k=\frac{2 \pi}{\hbar}\left|H_{D A}\right|^{2}(\mathrm{FC}),
$$

where $(\mathrm{FC})$ is the Franck-Condon factor, and $H_{D A}$ is the effective electronic coupling. Various approaches for treating the electronic coupling matrix element $H_{D A}$ have provided estimates of the decay coefficient. ${ }^{1-5,11,12}$ Separability of the electronic and nuclear factors is assumed in Eq. (1).

The effective coupling element can be defined as the coupling between the eigenstate $\left|\psi_{D}\right\rangle$ and the zeroth-order state $\left|\phi_{A}\right\rangle$, namely, $\left\langle\psi_{D}|H| \phi_{A}\right\rangle$. Using the partitioning technique ${ }^{13}$ it can be shown that this definition of effective coupling is the off-diagonal element after mapping the overall Hamiltonian onto an effective $2 \times 2$ Hamiltonian matrix of donor and acceptor states only. In an equivalent approach, a transfer operator $T$ can be defined from scattering theory ${ }^{14}$

$$
T=V+V G V,
$$

where $G$ is the Green's function for the Hamiltonian $H$. The latter is composed of an unperturbed $H^{0}$ and a perturbation $V$, where $H^{0}$ is the Hamiltonian for non-interacting donor, bridge and acceptor states, and $V$ is the interaction among them. By making use of the Lippman-Schwinger equation, it can be shown ${ }^{14}$ that the matrix element $\left\langle\phi_{D}|T| \phi_{A}\right\rangle$ is the same as the effective coupling $\left\langle\psi_{D}|H| \phi_{A}\right\rangle$, and the latter is denoted $H_{D A}$ throughout this article.

McConnell gave an early molecular derivation of the exponential decay factor. ${ }^{12} \mathrm{He}$ showed that for a single-band problem, where there is only one orbital per bridge site, the tight-binding Hamiltonian is tri-diagonal and the effective coupling matrix element $H_{D A}$ for a bridge with $n$ repeating units is

$$
H_{D A} \propto\left(\frac{\beta}{E-\alpha}\right)^{n},
$$

when $|E-\alpha| \gg 2|\beta|$. Here, $\beta$ is the interaction between neighboring orbitals, $\alpha$ is the energy of an individual orbital in the bridge, and $E$ is the energy of the electron to be transferred, namely, the energy of the donor orbital, which, in turn, equals the energy of the acceptor orbital when the system is at the transition state of the reaction. 
Further studies on the single-band case have appeared recently. ${ }^{2,3}$ Their analytic expression for $H_{D A}$ can be written as follows:

$$
H_{D A}=\frac{\beta_{A} \beta_{D}(-\beta)^{n-1} 2^{n+1} \zeta}{(E-\alpha+\zeta)^{n+1}-(E-\alpha-\zeta)^{n+1}},
$$

where

$$
\zeta=\left((E-\alpha)^{2}-4 \beta^{2}\right)^{1 / 2},
$$

where $n, E, \alpha$ and $\beta$ have the same definition as in Eq. (3), and $\beta_{D}\left(\beta_{A}\right)$ is the interaction matrix element between donor(acceptor) and the bridge unit it is attached to. In Eq. (4) it has been assumed that the basis formed by orbitals of donor and acceptor and orbitals on every site of bridge is orthonormalized. Otherwise one can always find a new set of basis by the transformation similar to that described in Ref. 15.

There are two cases when the expression in Eq. (4) tends to be exponential. One is for far off-resonance, namely,

$$
|E-\alpha| \gg 2|\beta| \text {. }
$$

Then, $\zeta$ is very close to $|E-\alpha|$ and so one of the two terms in the denominator vanishes under this condition. In this case McConnell's expression (Eq. (3)) is obtained. The other case is from the observation that the absolute values of the two terms in the denominator of Eq. (4) differ when $\zeta$ is a nonzero real number, which requires that

$$
|E-\alpha|>2|\beta| .
$$

This condition, together with the condition that $n$ be large, is a weaker condition on the energy of the electron or on the coupling strength. The inequality (7) also serves, for large $n$, as the off-resonance condition, since a tight-binding Hamiltonian of an infinite chain with one orbital per site has an energy band which lies between $\alpha-2 \beta$ and $\alpha+2 \beta$. If Eq. (7) holds and $n$ is large, the denominator of Eq. (4) is dominated by one of the two terms that has larger absolute value for the case that $n$ is large. Consequently, exponential behavior is obtained in the limit of long chain bridges, where the attenuation factor is close to, but not exactly the same as the $\beta /(E-\alpha)$ in Eq. (3).

Beratan and Hopfield ${ }^{4}$ used another approach with which they were able to treat more realistic systems, i.e. severalband systems. Their method is readily understood if we note that surface states exist when the energy of the surface atom (donor/acceptor orbitals) lies outside the energy band of the infinite chain(bridge). ${ }^{15}$ The usual Bloch states have complex eigenvalues with unit moduli for the translation operator that commutes with the Hamiltonian of the infinite chain, while such surface states have real eigenvalues for the translation operator. Since the wavefunctions must be square-integrable, the wavefunction for a surface state must be decaying rather than growing exponentially as it penetrates the infinitely long bridge. The energy and the corresponding decaying factor were solved by fitting the boundary condition at each end. Thereby, the exponential behavior was built into their solution. As discussed above, for a single band bridge, the expo- nential dependence is found either for off-resonant, sufficiently long uniform bridge chains or for energies far moved from the bridge band.

In the present paper we consider a more general case, which is not limited to systems with a single orbital in each bridge site or to a large system. For convenience and simplicity, the term "band" will be used in a loose sense and refers to the region where energy levels are concentrated or where the actual band of an infinite chain would be, even though we will be discussing finite systems only. In Section II, a sequential expression for the Green's function is obtained. The Green's function of $n$ bridge units is written in terms of that for $n-1$ units. The derivation does not require that the bridge units be identical, and they are also allowed to have different numbers of orbitals. In Section III, this method is applied to calculating the electron transfer rate between an electro-active group on an adsorbed alkane thiol molecule and the electrode to which it is attached. The effect of additional parallel chains of alkane thiol molecules was also treated and, together with the comparison between our sequential method and the direct summation over the bridge eigenstates, the results are discussed in Section IV. Concluding remarks are given in Section V. The Appendix consists of the graphical derivation of the sequential formula and its possible generalization.

\section{THE SEQUENTIAL FORMULA FOR GREEN'S FUNCTION}

The tight-binding Hamiltonian is considered with only the nearest-neighbor interaction, namely, between neighboring bridge units, between the first unit and the donor orbital, and between the last unit and the acceptor. An expression for the Green's function for the whole space by the method provided below can always be developed, but the expression for the bridge Green's function is much simpler to introduce and for off-resonant systems it provides a satisfactory approximation. Thereby, it is assumed here that the matrix elements of the Green's function needed in Eq. (2) are approximately those of the Green's function for the bridge part only. The error from such an approximation should usually be relatively small when compared to other approximations made in the tight-binding calculation.

It is always possible to calculate the Green's function for short bridge chains, given the explicit Hamiltonian matrix elements. Therefore, we have explored solving the problem for general chain lengths, assuming that the Green's function is known for a chain with one less bridge unit and then, for longer bridge chains, obtaining the recursion equations and iterating them until the desired length. This iteration process involves mostly matrix multiplication and inversion. All the matrices involved will be seen to have dimensions determined by the number of molecular orbitals on each related bridge unit. These numbers are finite and are independent of the number of units of the entire bridge, and so the iteration process for longer bridge chains can be executed without solving a large linear problem. For notational simplicity, the derivation is given for systems with uniform bridge units. 
The generalization to arbitrary different bridge units can be made without difficulty and is discussed in Section IV. The derivation given below for the bridge's Green's function is non-perturbative with respect to the magnitude of the intrabridge interactions.

It is supposed here that there are $n$ bridge units in the problem, and that each bridge unit has $m$ molecular orbitals. Using the basis that diagonalizes the Hamiltonian within each bridge unit, ${ }^{16}$ the Hamiltonian for a chain bridge is

$$
H^{(n)}=\left(\begin{array}{ccccc|c}
\mathbf{e} & \mathbf{v} & \mathbf{0} & \mathbf{0} & \cdots & \mathbf{0} \\
\mathbf{v}^{T} & \mathbf{e} & \mathbf{v} & \mathbf{0} & \cdots & \vdots \\
\mathbf{0} & \mathbf{v}^{T} & \mathbf{e} & \mathbf{v} & \cdots & \vdots \\
\vdots & \vdots & \vdots & \vdots & \ddots & \mathbf{v} \\
\hline \mathbf{0} & \cdots & \cdots & \mathbf{0} & \mathbf{v}^{T} & \mathbf{e}
\end{array}\right)
$$

where $\mathbf{e}$ is an $m \times m$ diagonal matrix

$$
\mathbf{e}=\left(\begin{array}{cccc}
\varepsilon_{1} & 0 & \cdots & 0 \\
0 & \varepsilon_{2} & \cdots & 0 \\
\vdots & \vdots & \ddots & \vdots \\
0 & 0 & \cdots & \varepsilon_{m}
\end{array}\right),
$$

and $\mathbf{v}^{T}$ is the transpose of the interaction matrix $\mathbf{v}$ that couples adjacent bridge units. The lines in Eq. (8) partition the matrix into four blocks. The upper left one, a large square block, corresponds to the Hamiltonian for $n-1$ bridge units. The elements in the two off-diagonal blocks arise as a perturbation designated below as $\mathscr{H}_{1}^{(n)}$. The elements in the two diagonal blocks form the zeroth-order Hamiltonian $\mathscr{H}_{0}^{(n)}$. Thereby, $\mathscr{H}_{0}^{(n)}$ and $\mathscr{H}_{1}^{(n)}$ are defined as

$$
\begin{aligned}
\mathscr{H}_{0}^{(n)} & =\left(\begin{array}{ccccc|c}
\mathbf{e} & \mathbf{v} & \mathbf{0} & \mathbf{0} & \cdots & \mathbf{0} \\
\mathbf{v}^{T} & \mathbf{e} & \mathbf{v} & \mathbf{0} & \cdots & \vdots \\
\mathbf{0} & \mathbf{v}^{T} & \mathbf{e} & \mathbf{v} & \ldots & \vdots \\
\vdots & \vdots & \vdots & \vdots & \ddots & \mathbf{0} \\
\hline \mathbf{0} & \cdots & \cdots & \mathbf{0} & \mathbf{0} & \mathbf{e}
\end{array}\right) \\
& \equiv\left(\begin{array}{c|c}
H^{(n-1)} & \mathbf{0} \\
\hline \mathbf{0} & \mathbf{e}
\end{array}\right),
\end{aligned}
$$

and

$$
\mathscr{H}_{1}^{(n)}=\left(\begin{array}{ccccc|c}
\mathbf{0} & \mathbf{0} & \mathbf{0} & \mathbf{0} & \cdots & \mathbf{0} \\
\mathbf{0} & \mathbf{0} & \mathbf{0} & \mathbf{0} & \ldots & \mathbf{0} \\
\mathbf{0} & \mathbf{0} & \mathbf{0} & \mathbf{0} & \ldots & \mathbf{0} \\
\vdots & \vdots & \vdots & \vdots & \ddots & \mathbf{v} \\
\hline \mathbf{0} & \cdots & \cdots & \mathbf{0} & \mathbf{v}^{T} & \mathbf{0}
\end{array}\right),
$$

so that $H^{(n)}=\mathscr{H}_{0}^{(n)}+\mathscr{H}_{1}^{(n)}$. The $\mathscr{H}_{0}^{(n)}$ is seen in Eq. (10) to refer to a fully coupled $(n-1)$-unit bridge plus an uncoupled $n$th bridge unit attached.

The Green's function corresponding to the $H^{(n)}$ in Eq. (8) is then rewritten as

$$
\begin{aligned}
G^{(n)} & =\left(E \mathbf{1}-H^{(n)}\right)^{-1}=\left(E \mathbf{1}-\mathscr{H}_{0}^{(n)}-\mathscr{H}_{1}^{(n)}\right)^{-1} \\
& =\mathscr{G}_{0}^{(n)}\left(\mathbf{1}-\mathscr{H}_{1}^{(n)} \mathscr{G}_{0}^{(n)}\right)^{-1},
\end{aligned}
$$

where $G^{(n)}$ is the Green's function for the tight-binding $n$-unit bridge system, and $\mathscr{G}_{0}^{(n)}$ is the Green's function corresponding to $\mathscr{H}_{0}^{(n)}$ :

$$
\mathscr{G}_{0}^{(n)}=\left(E \mathbf{1}-\mathscr{H}_{0}^{(n)}\right)^{-1}=\left(\begin{array}{c|c}
G^{(n-1)} & \mathbf{0} \\
\hline \mathbf{0} & \Delta^{-1}
\end{array}\right),
$$

here $\Delta$ denotes the diagonal $m \times m$ matrix

$$
\Delta=E \mathbf{1}-\mathbf{e} .
$$

The term $\left(\mathbf{1}-\mathscr{H}_{1}^{(n)} \mathscr{G}_{0}^{(n)}\right)$ can be written as

$$
\mathbf{1}-\mathscr{H}_{1}^{(n)} \mathscr{G}_{0}^{(n)}=\left(\begin{array}{c|c}
\mathbf{1}_{m(n-1)} & -M_{2} \\
\hline-M_{1} & \mathbf{1}_{m}
\end{array}\right),
$$

where $\mathbf{1}_{m(n-1)}$ and $\mathbf{1}_{m}$ denote unit square matrices of dimensions given by subscript. The $M_{1}$ and $M_{2}$ are given by

$$
M_{1}=\left(\begin{array}{llll}
\mathbf{v}^{T} G_{(n-1,1)}^{(n-1)} & \mathbf{v}^{T} G_{(n-1,2)}^{(n-1)} & \cdots & \left.\mathbf{v}^{T} G_{(n-1, n-1)}^{(n-1)}\right),
\end{array}\right.
$$

and

$$
M_{2}=\left(\begin{array}{c}
\mathbf{0} \\
\vdots \\
\mathbf{0} \\
\mathbf{v} \Delta^{-1}
\end{array}\right),
$$

in which $\mathbf{v}, \mathbf{v}^{T}, \Delta^{-1}$ and $G_{(i, j)}^{(n-1)}$ all represent $m \times m$ matrices. Specifically, $G_{(n-1,1)}^{(n-1)}$ is the $(n-1,1)$ block in the Green's function $G^{(n-1)}$ for $n-1$ bridge units. The inverse of the matrix in Eq. (15) can be written as

$$
\begin{aligned}
\left(\mathbf{1}-\mathscr{H}_{1}^{(n)} \mathscr{G}_{0}^{(n)}\right)^{-1}= & \left(\begin{array}{c|c}
\mathbf{1}_{m(n-1)} & M_{2} \\
\hline M_{1} & \mathbf{1}_{m}
\end{array}\right) \\
& \times\left(\begin{array}{c|c}
\left(\mathbf{1}-M_{2} M_{1}\right)^{-1} & \mathbf{0} \\
\hline \mathbf{0} & \left(\mathbf{1}-M_{1} M_{2}\right)^{-1}
\end{array}\right),
\end{aligned}
$$

as can be verified by multiplying both sides of Eq. (15) to the left or to the right with the matrix in Eq. (18). Since there is negligible direct coupling between the donor and acceptor states in the long-range electron transfer, the effective coupling is calculated from the second term in Eq. (2). Also because the tight-binding model is used, only one block of the Green's function $G^{(n)}$ is needed in that expression, namely, the block relating transition from the first bridge unit to the $n$th one. It is denoted by $G_{(1, n)}^{(n)}$ and is a block of dimensions $m \times m$. The Green's function $G^{(n)}$ is obtained by introducing Eqs. (13) and (18) into Eq. (12) and performing the matrix multiplication in terms of blocks. For the desired $(1, n)$ block, we obtain

$$
G_{(1, n)}^{(n)}=G_{(1, n-1)}^{(n-1)} \mathbf{v} \Delta^{-1}\left(\mathbf{1}-\mathbf{v}^{T} G_{(n-1, n-1)}^{(n-1)} \mathbf{v} \Delta^{-1}\right)^{-1} .
$$


In order to iterate Eq. (19) further for $G_{(1, n+1)}^{(n+1)}$, an expression for $G_{(n, n)}^{(n)}$ is needed. This $m \times m$ block matrix can be obtained similarly from $G^{(n)}$ but selecting the $(n, n)$ block,

$$
G_{(n, n)}^{(n)}=\Delta^{-1}\left(\mathbf{1}-\mathbf{v}^{T} G_{(n-1, n-1)}^{(n-1)} \mathbf{v} \Delta^{-1}\right)^{-1} .
$$

Equation (20) is a recursion expression for $G_{(n, n)}^{(n)}$, However, it would be desirable to convert the expression to one in which a dimensionless quantity represents the deviation from the first-order term in the expansion of Eq. (12). ${ }^{17}$ With this goal in mind, we define an $m \times m$ block $N_{n}$,

$$
N_{n}=\Delta G_{(n, n)}^{(n)} .
$$

A recursion expression for $N_{n}$ then follows from Eq. (20):

$$
N_{n}=\left(\mathbf{1}-\mathbf{v}^{T} \Delta^{-1} N_{n-1} \mathbf{v} \Delta^{-1}\right)^{-1} .
$$

Equation (19) then becomes

$$
G_{(1, n)}^{(n)}=G_{(1, n-1)}^{(n-1)} \mathbf{v} \Delta^{-1} N_{n} .
$$

The initial condition for the iteration of $\left\{N_{n}\right\}$ is taken as $N_{2} ; N_{2}$ can be obtained by inverting the $2 m \times 2 m$ tightbinding Hamiltonian, together with Eq. (21). Since the above derivation does not introduce any explicit assumption that $\mathbf{v} \Delta^{-1}$ is small, i.e. Eqs. (22) and (23) were not derived perturbatively, Eqs. (22) and (23) are mathematically exact for finite $n$.

The factor $\mathbf{v} \Delta^{-1}$ in Eq. (23) resembles McConnell's estimate of the scalar decay factor $\beta /(E-\alpha)$ (Eq. (3)). If the matrix $N_{n}$ becomes essentially a constant matrix after a number of iterations, then the overall trend for $G_{(1, n)}^{(n)}$ is to become an exponential as $n$ increases. The sequential formula of $N_{n}$ (Eq. (22)) is a non-linear first order difference equation for matrices.

It is instructive, for understanding the general properties of Eq. (22), to examine the solution of this difference equation for $m=1$, where $N_{n}$ becomes a scalar. In this case, $N_{n}$ can now be solved by the transformation:

$$
a_{n}=\frac{1-2 r^{2} N_{n}}{\sqrt{\left|1-4 r^{2}\right|}},
$$

so Eq. (22) yields

$$
\frac{a_{n}-a_{n-1}}{a_{n} a_{n-1} \pm 1}=\sqrt{\left|1-4 r^{2}\right|},
$$

where $r$ is defined as the scalar $\mathbf{v} \Delta^{-1}(\equiv \beta /(E-\alpha))$ and the negative sign is taken if $|r|<1 / 2$ and the positive sign, otherwise. Equation (24) can be solved by writing $a_{n}$ as $\tanh b_{n}\left(\right.$ or $\left.\tan b_{n}\right)$ and making use of the addition formula of the hyperbolic tangent (or tangent) function. The following solution is then obtained:

$$
\begin{aligned}
N_{n}= & \frac{1}{2 r^{2}}-\frac{\sqrt{1-4 r^{2}}}{2 r^{2}} \tanh \left(\tanh ^{-1} \frac{\left(1-2 r^{2}\right) N_{2}}{\sqrt{1-4 r^{2}}}\right. \\
& \left.+(n-2) \sqrt{1-4 r^{2}}\right), \text { if }|r|<\frac{1}{2} ;
\end{aligned}
$$

$$
\begin{aligned}
N_{n}= & \frac{1}{2 r^{2}}-\frac{\sqrt{4 r^{2}-1}}{2 r^{2}} \tan \left(\tan ^{-1} \frac{\left(1-2 r^{2}\right) N_{2}}{\sqrt{4 r^{2}-1}}\right. \\
& \left.+(n-2) \sqrt{4 r^{2}-1}\right), \text { if }|r|>\frac{1}{2},
\end{aligned}
$$

with

$$
N_{2}=\frac{1}{1-r^{2}} \text {. }
$$

In the former case, $N_{n}$ approaches a constant as $n$ increases, while in the latter case, $N_{n}$ has an oscillating behavior arising from equally spaced poles on the real $n$-axis. Basically this result is the solution of a single-band problem. When compared to Eq. (4), Eqs. (23) and (25) give the same exponential factor for the off-resonance case. It should be noted that the bridge Green's function was used here, while in obtaining Eq. (4) the full tight-binding Hamiltonian, including donor and acceptor states, is used instead. The general solution of Eq. (23) for multi-band Green's function $\left\{G_{(1, n)}^{(n)}\right\}$, was also obtained without iteration, but the solution we obtained involves the inverse of the sum of the $(n-3)$ th power of two matrices for $n \geqslant 3$, making it numerically unstable to calculate when $n$ is large in its present form. The result is given in Ref. 18. Practically, the sequential formula, Eq. (22), is straightforward and stable to use.

By observing the result of numerical iteration, the behavior of $N_{n}$ can be described for most cases. As in the corresponding single-band case, the matrix $N_{n}$ tends to a constant matrix if the energy $E$ is outside all the bridge " 'bands.' ' In this case, all of the eigenvalues of $\mathbf{v} \Delta^{-1} N_{n}$ lie inside the unit circle of the complex plane and one or two of them dominate the final decay factor as $n$ becomes large, namely the one with the largest modulus. If there is only one dominating eigenvalue, an exponential decay in the coupling strength with respect to $n$ would be obtained. If the eigenvalues with the largest modulus are a pair of complex conjugate numbers the decay is modified by a periodic oscillation. For the cases where $E$ lies inside the bridge bands, Eqs. (22) and (23) are still mathematically applicable with a complicated dependence of $N_{n}$ and $G_{(1, n)}^{(n)}$ on $n$, but physically the assumption of approximating the overall Green's function with the Green's function for the bridge part is not a good one. Therefore, it is inappropriate to discuss the onresonance condition using the sequential formula in its present form.

We have also obtained a graph-based method of deriving Eqs. (22) and (23) which is potentially useful for complicated bridge systems. It is outlined in the Appendix. In this graph-based method, an infinite series is obtained, but its summation yields the same equation as that obtained in the above derivation. Since the latter did not involve any infinite series, it is seen that analytical continuation of the series can be used to obtain valid results in a region where the original infinite series of the graph-based method diverges. This property, if it still holds in more complicated problems, en- 
larges the scope of the graph-based method beyond its infinite series approach, permitting its use in other applications.

\section{APPLICATION}

The recent development of self-assembled monolayers of alkane thiol molecules on a gold surface has provided a convenient approach for studying electron transfer between an electroactive group and an electrode, where the electroactive group is held at a fixed distance from electrode surface, or, if in solution, is separated from the electrode by a fixed monolayer. ${ }^{6}$ Chidsey measured the voltage dependent ET rate of the ferrocenylcarboxy-terminated alkane thiol/gold surface system including the rate under the exchange current condition (corresponding to the case where the $\Delta G^{0}$ for the electrode process is zero) and a reorganization energy $\lambda$ was estimated by fitting the data to an equation whose functional form is similar to Eq. (27) below. ${ }^{7}$

A study involving electrodes coated with self-assembly monolayers of $\omega$-hydroxy thiols of various lengths (the $n$ 's of $\left(-\mathrm{CH}_{2}-\right)$ are 6 to 11$)$ was performed by Becka and Miller. ${ }^{8}$ The electron transfer current of anions in solution was measured and after corrections for diffusion and other effects were made, the authors reported the length dependence factor $\beta=1.08 \pm 0.20$ per methylene unit. ${ }^{19}$ Carter et $a l .{ }^{9}$ studied, for different lengths of thiol molecules ( $n=8,12,16)$, the system investigated by Chidsey. ${ }^{7}$ The length dependence over the above range was obtained and the decay factor was reported as $\beta=1.44 \pm 0.12$ per methylene unit.

\section{A. The nonadiabatic reaction rate}

A mathematical form for the nonadiabatic rate between an electron donor group and an electrode, in the high temperature limit, is given by ${ }^{20,21}$

$$
\begin{aligned}
k_{\text {rate }}= & \frac{2 \pi}{\hbar}\left(4 \pi \lambda k_{B} T\right)^{-1 / 2} \\
& \times \int d \varepsilon|V(\varepsilon)|^{2} e^{-(\lambda-e \eta+\varepsilon)^{2} / 4 \lambda k_{B} T} \frac{e^{\varepsilon / k_{B} T}}{1+e^{\varepsilon / k_{B} T}},
\end{aligned}
$$

where $|V(\varepsilon)|^{2}$ is

$$
|V(\varepsilon)|^{2}=\int d^{3} \vec{k}\left|H_{D k}\right|^{2} \delta(\varepsilon(\vec{k})-\varepsilon),
$$

in which $H_{D k}$ is used to denote the effective coupling element between states $\langle D|$ and $|\vec{k}\rangle$, with the definition similar to that of $H_{D A}$ described in Sec. I, the wavefunctions $|\vec{k}\rangle$ are normalized to a Dirac delta function, $\left\langle\vec{k} \mid \vec{k}^{\prime}\right\rangle=\delta\left(\vec{k}-\vec{k}^{\prime}\right)$, $\varepsilon$ is the energy of an electron in metal with respect to the Fermi energy, $\varepsilon(\vec{k})$ is the energy of the electronic state $|\vec{k}\rangle$ of the metal, $\lambda$ is the reorganization energy (including both inner and outer contribution), and $\eta$ is the overpotential $\left(E-E^{0}\right)$, namely the difference between the applied potential and the standard potential of the electrode. The above Eq. (27) is an integral of the non-adiabatic electron transfer rate expression ${ }^{22}$ over all the possible states $|\vec{k}\rangle$ and all possible energies $\varepsilon$ in a metal, using the Fermi-Dirac distribu- tion as a weighting factor. In general, a summation over energy bands of the metal electrode is needed in Eq. (28). However, since in the present work we only consider the contribution from the $s$-band of the gold electrode, the summation over energy bands is omitted for simplicity. "Work terms ${ }^{20}$ are also omitted in Eq. (27) for simplicity of notation. One sees that because of the delta function normalization and the above definition of $H_{D k}$, the $|V(\varepsilon)|^{2}$ in Eq. (28) has units of energy.

The electron transfer rate under electrochemical exchange current conditions (i.e., forward rate equal to reverse rate) can be obtained by setting $\eta=0$. By noting $\lambda \gg \varepsilon$ we can drop the quadratic term of $\varepsilon$ in the exponent of Eq. (27). The integration over $\varepsilon$ is performed by approximating 20

$$
\begin{aligned}
\int_{-\infty}^{\infty} & \frac{1}{e^{\varepsilon / 2 k_{B} T}+e^{-\varepsilon / 2 k_{B} T} f(\varepsilon) d \varepsilon} \\
& \equiv \int_{-\infty}^{\infty} g(\varepsilon) f(\varepsilon) d \varepsilon \\
& =f(0) \int_{-\infty}^{\infty} g(\varepsilon) d \varepsilon+f^{\prime}(0) \int_{-\infty}^{\infty} g(\varepsilon) \varepsilon d \varepsilon+\ldots \\
& \approx \pi k_{B} T f(0),
\end{aligned}
$$

since $g(\varepsilon)$ here is a symmetric, positive definite function with its weight concentrated around the origin. From Eqs. (27) and (29), the following expression is obtained:

$$
k_{\text {rate }}^{(\mathrm{ex})}=\frac{2 \pi}{\hbar}\left(4 \pi \lambda k_{B} T\right)^{-1 / 2} e^{-\lambda / 4 k_{B} T}|\bar{V}|^{2},
$$

where

$$
|\bar{V}|^{2}=\pi k_{B} T \int d^{3} \vec{k}\left|H_{D k}\right|^{2} \delta(\varepsilon(\vec{k}))=\pi k_{B} T \overline{\left|H_{D k}\right|^{2}} \rho_{f},
$$

with

$$
\overline{\left|H_{D k}\right|^{2}} \equiv \frac{\int d^{3} \vec{k}\left|H_{D k}\right|^{2} \delta(\varepsilon(\vec{k}))}{\int d^{3} \vec{k} \delta(\varepsilon(\vec{k}))}
$$

and

$$
\rho_{f} \equiv \int d^{3} \vec{k} \delta(\varepsilon(\vec{k}))
$$

Namely, $\overline{\left|H_{D k}\right|^{2}}$ is the effective coupling strength averaged over the $\vec{k}$ 's on the Fermi surface and has units of

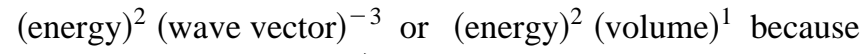
of the normalization of $|\vec{k}\rangle$ described earlier, while $\rho_{f}$ is the density of states at the Fermi surface, with units of (energy) $^{-1}$ (wave vector) $^{3}$. In the present work, the unit length is chosen to be the nearest-neighbor distance of the fcc lattice of $\mathrm{Au}$ atoms, and so (wave vector) $^{3}$ equals (number of atoms) $)^{-1}$.

To calculate theoretically the effective coupling matrix element $H_{D k}$ between the ferrocenylcarboxyl group and the 
gold electrode and compare with the experimental data, two of the schemes, (a) and (b), which are developed from Eq. (2), assuming no direct coupling, are listed.

(a) A direct summation over all bridge molecular orbitals can be made by using the following expression:

$T_{D k}\left(\equiv H_{D k}\right)=\sum_{B} \frac{V_{D B} V_{B k}}{E-E_{B}}$,

where $\{B\}$ denotes the set of molecular orbitals of the bridge. It can be shown that the matrix element $T_{D k}$ of the transfer operator equals the effective coupling $H_{D k},{ }^{14}$ as discussed in Section I. The bridge Green's function is used to replace the overall Green's function. Also, the perturbation $V$ is regarded as the interaction of any state of the entire bridge with the donor and with the acceptor. Namely $V_{D B}$ is the interaction matrix element between the donor state and the orbital $B$ of the entire bridge.

(b) The sequential formula derived in Section II (Eqs. (22) and (23)), can be used:

$H_{D k}=V_{D, 1} G_{(1, n)}^{(n)} V_{n, k}$,

where $V_{D, 1}$ and $V_{n, k}$ denote interaction between donor state and the molecular orbitals in the first bridge unit and between those in the $n$th bridge unit and the state $|\vec{k}\rangle$ of the metal electrode, respectively. For a bridge with more than one orbital on each site, $V_{D, 1}$ denotes a row vector, $G_{(1, n)}^{(n)}$ a matrix, and $V_{n, k}$ a column vector.

In both cases, the wavefunctions used for the $\mathrm{Au}(111)$ surface are linear combinations of atomic $s$-orbitals obtained with the tight-binding approximation, ${ }^{23}$ and to evaluate Eq. (31), the coupling strength of 60 wave vectors $(\vec{k})$ random sampled over the Fermi surface were calculated and averaged to obtain $\overline{\left|H_{D k}\right|^{2}}$. All of the interaction matrix elements were obtained using an extended Hückel program. ${ }^{24}$ The coordinates of atoms in the alkane thiol portion of the system are those of Klein and coworkers, who employed a molecular dynamics calculation in conjunction with structural data. ${ }^{25,26}$ The geometry of the ferrocenylcarboxyl group is obtained from the crystal structure of similar molecules. ${ }^{27} \mathrm{In}$ the supplemental material ${ }^{28}$ we deposited the full Cartesian coordinates for the molecules being used in the present work.

\section{B. The energy difference at transition state}

To calculate the denominator of Eq. (32) or the Green's function $G_{(1, n)}^{(n)}$ of Eq. (33), it is necessary to know the energy of the various electronic states of the bridge $(B)$ relative to the Fermi level of the metal $(M)$. We consider the free energy $v s$. the reaction coordinate $q$ diagram in Fig. 1, which describes the reaction which involves transfer of an electron from $D$ to a specific orbital $|\vec{k}\rangle$ at the Fermi energy in the metal, ${ }^{21}$

$$
D+B+M \rightarrow D^{+} \text {(solvated) }+B+M(e) .
$$

Curves I and II describe the left side and the right side of Eq. (34) respectively. Curve III corresponds to the superexchange state denoted by $D^{+}+B^{-}$(unsolvated) $+M$, if it is

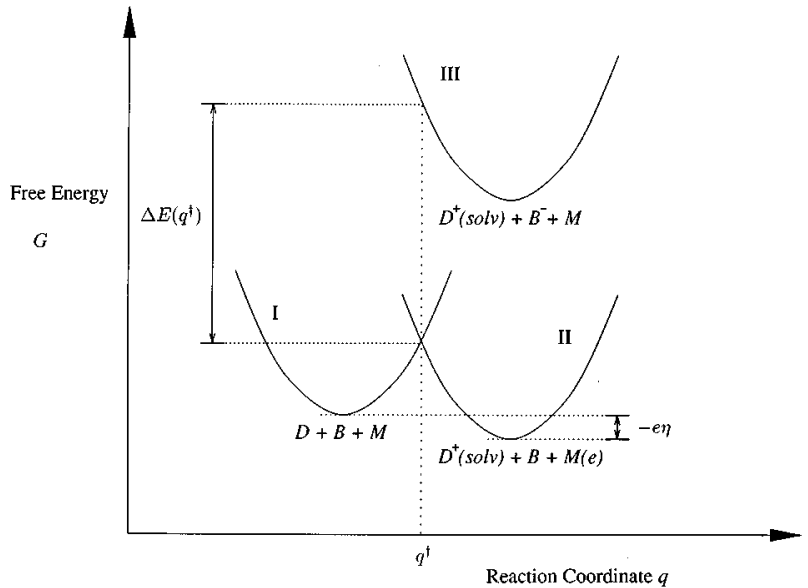

FIG. 1. Free energy vs. reaction coordinate for the reactant (curve I) and product (curve II) states of the bridge-mediated electron transfer reaction at an electrode. Curve III is the superexchange off-resonant state for an electron transfer scheme. $D$ is the donor molecule, a ferrocenylcarboxyl group in the present case. $\Delta E\left(q^{\dagger}\right)$ is the energy difference of curves I and III at the transition state.

an electron transfer (For a hole transfer, a curve representing $D+B^{+}$(unsolvated) $+M(e)$ should be used instead.) The energetics for I and II for the free energy $G(q)$ as a function of $q$ are described by:

$$
\begin{aligned}
& G_{\mathrm{I}}(q)=G_{D}(q)+E_{D}, \\
& G_{\mathrm{II}}(q)=G_{D^{+}}(q, \text { solv })+\bar{\mu}_{m}+e \phi_{s},
\end{aligned}
$$

where $E_{D}$ is the energy of the electronic orbital of donor $D$ with respect to vacuum, $G_{D}(q)$ is the solvation free energy of $D$, as a function of $q$, and $G_{D^{+}}(q$, solv $)$ is similarly the solvation free energy of $D^{+} ; \bar{\mu}_{m}$ is the electrochemical potential (Fermi level) of an electron in $M$, and is equal to $\mu_{m}^{0}-e \phi_{m}$. The $\phi$ 's denote electrostatic potentials (the socalled inner electric potential or the Galvani potential ${ }^{29,30}$ ) for the metal eletrode $\left(\phi_{m}\right)$ and in solution $\left(\phi_{s}\right)$.

The bridge $B$ can become a $B^{+}$(or a $B^{-}$) in the virtual state which occurs in the superexchange mechanism, but because of the off-resonance condition this supertransient $B^{+}$ or $B^{-}$can be regarded as unsolvated. There may be some interaction of this virtual electronic state with the electrons in the surrounding medium, but we will neglect such details here. If the potential change $\phi_{m}-\phi_{s}$ occurs across the adsorbed monolayer $B$, then a first approximation would be to treat the energy levels of $B$ as being at a mean electrostatic potential $\left(\phi_{m}+\phi_{s}\right) / 2$. In that case we have, for the electrontransfer scheme,

$$
G_{\mathrm{III}}\left(q, B_{i}\right)=G_{D^{+}}(q, \text { solv })+E_{B i}+e \phi_{s}-\frac{e}{2}\left(\phi_{m}+\phi_{s}\right),
$$

where $E_{B i}$ is the energy of the $i$ th orbital of the bridge in the absence of an electrostatic potential.

The vertical difference between I and III at the transition state is denoted by $\Delta E\left(q^{\dagger}\right)$ in Fig. 1. It is seen from Eqs. (35) and (37) to be 


$$
\begin{aligned}
\Delta E\left(q^{\dagger}\right) & =G_{\mathrm{I}}\left(q^{\dagger}\right)-G_{\mathrm{III}}\left(q^{\dagger}\right)=G_{\mathrm{II}}\left(q^{\dagger}\right)-G_{\mathrm{III}}\left(q^{\dagger}\right) \\
& =\mu_{m}^{0}-E_{B i}-\frac{e}{2}\left(\phi_{m}-\phi_{s}\right) \\
& =\mu_{m}^{0}-E_{B i}-\frac{e}{2}\left(\phi_{m}^{0}-\phi_{s}^{0}+\eta\right),
\end{aligned}
$$

since $G_{\mathrm{I}}=G_{\mathrm{II}}$ in the transition state, $q=q^{\dagger}$. In Eq. (40) the over-potential $\eta$ has been defined as $\phi_{m}-\phi_{s}-\phi_{m}^{0}+\phi_{s}^{0}$ while $\left(\phi_{m}^{0}-\phi_{s}^{0}\right)$ is the standard metal-solution potential difference of the electrode. From Fig. 1 and Eq. (38), $\Delta E\left(q^{\dagger}\right)$ is seen to be independent of the reorganizational energy $\lambda$ since the solvational free energies of the intermediate state (III) and the final state (II) are the same (for $D^{+}$ion) and so the reorganizational energy cancels in the $G_{\mathrm{I}}\left(q^{\dagger}\right)-G_{\mathrm{III}}\left(q^{\dagger}\right)$ difference. $\eta$ equals zero for the exchange current condition.

In Eq. (39) both $\mu_{m}^{0}$ and $E_{B i}$ are negative quantities that describe the energy required to move the electron from the neutral materials to vacuum at infinity, while $-e \phi_{m}$ and $-e\left(\phi_{m}+\phi_{s}\right) / 2$ adjust that energy for the effect of the electric potential. This process of moving an electron to vacuum at infinity can also be described as occurring in the following two steps: the electron is first moved to just outside the surface of the material in vacuum, and then it is moved from that point to infinity. The energy needed in the first step is the definition of the work function of the material, ${ }^{31}$ and that for the second step is the electronic charge times the outer potential $\psi$ (termed also the Volta potential), ${ }^{29,32}$ namely, the electric potential of the material due to its total charge. The inner potential $\phi$ 's and outer potential $\psi$ 's differ by a surface term which is due to the dipolar distribution of charge at the surface of the material. If the work function of the metal is denoted $\Psi_{m}$ (a positive quantity), and the corresponding quantity for the bridge molecule is $\Psi_{B}$, the ionization potential, we have the following relations for metal and bridge respectively, equating the two ways of accounting for the energy of the electron in the material relative to its value when at rest in vacuum at infinity:

$$
\begin{aligned}
& \mu_{m}^{0}-e \phi_{m}=-\Psi_{m}-e \psi_{m}, \\
& E_{B}(\mathrm{HOMO})-\frac{e}{2}\left(\phi_{m}+\phi_{s}\right)=-\Psi_{B}-\frac{e}{2}\left(\psi_{m}+\psi_{s}\right),
\end{aligned}
$$

and for other molecular states in $B$, the same energy difference $\Delta \epsilon_{i}\left(=E_{B i}-E_{B}(\mathrm{HOMO})\right)$ can be added to both sides of Eq. (42).

With Eqs. (41) and (42), Eq. (40) can be written as follows: (for the exchange current condition, the over-potential $\eta$ has been set to zero)

$$
\begin{aligned}
\Delta E\left(q^{\dagger}\right) & =-\Psi_{m}-e\left(\psi_{m}^{0}-\psi_{s}^{0}\right)+\Psi_{B}-\Delta \epsilon_{i}+\frac{e}{2}\left(\psi_{m}^{0}-\psi_{s}^{0}\right) \\
& =-e E_{(\mathrm{abs})}^{0}+\frac{e}{2}\left(\psi_{m}^{0}-\psi_{s}^{0}\right)+\Psi_{B}-\Delta \epsilon_{i},
\end{aligned}
$$

where $\psi^{0}$ denotes the corresponding outer potential when the potential of the electrode is at the standard potential of the redox species, and $E_{(\mathrm{abs})}=\Psi_{m} / e+\left(\psi_{m}-\psi_{s}\right)$ is the absolute electrode potential with its reference state being an electron at rest in vacuum close to the surface of the solution, as discussed by Trasatti in Refs. 30 and 32. The absolute potential for some of the commonly used reference electrodes are also listed in Ref. 32. With these data, the absolute potential for the electrode described by Chidsey ${ }^{7}$ is estimated to be $5.13 \mathrm{~V}$, if the standard potential $E^{0}$ is taken as $0.08 \mathrm{~V}$ above the $\mathrm{Ag} /\left(1 \mathrm{mM} \mathrm{AgClO} 4,1 \mathrm{M} \mathrm{HClO}_{4}\right)$ reference electrode. Together with the work function of the $\mathrm{Au}(111)$ surface, $5.31 \mathrm{eV},{ }^{33}$ we obtain $-0.18 \mathrm{~V}$ for the potential difference, $\psi_{m}^{0}-\psi_{s}^{0}$ With these quantities, Eq. (40) becomes

$$
\Delta E\left(q^{\dagger}\right)=-5.22 \mathrm{eV}+\Psi_{B}-\Delta \epsilon_{i}
$$

for the $i$ th molecular orbital of the bridge. $\Delta E\left(q^{\dagger}\right)$ is the quantity that is needed both in the denominator of Eq. (32) and the Green's function of Eq. (33). The quantity $-\Psi_{B}+\Delta \epsilon_{i}$ representing the energy of the $i$ th bridge state, is obtained in the following section.

\section{Energy of bridge states}

For use in both the summation and the sequential methods in the present calculation, the energy eigenvalues for a long alkane chain $\left(\left(\mathrm{CH}_{2}\right)_{n}, n=40\right.$ or more) were obtained from either the extended-Hückel or the tight-binding Hamiltonian, for comparison with the experimental data on the band structure of polyethylene. To obtain a better agreement with those data, adjustments of the Hamiltonians are given below. Such adjustments are then applied to each alkanethiol bridge in the calculation.

In the direct summation calculation, a full extendedHückel calculation was performed. As noted in Ref. 34, in describing the valence band structure of polyethylene, the extended-Hückel method itself does surprisingly well. By comparing the distribution of calculated energy levels (Fig. 2 , part I) with the experimental valence band structure, ${ }^{34}$ we concluded that the following adjustments were needed for the position of each bridge level (denoted by $E_{B}$ in Eq. (32)): first, a factor of 0.7 is used to multiply the energies of the levels in the filled extended-Hückel band formed from $\mathrm{C}$ $2 p$ and $\mathrm{H} 1 s$ orbitals (denoted by (a) in Fig. 2, part I) so that the bandwidth is closer to that given by experiment, and then the two valence bands ((a) and (b) in Fig. 2, part I) are shifted to fit the experimental band edges for each band. ${ }^{34}$ Prior to any adjustment, the position of the lower edge of band (c) is calculated to be about $-0.6 \mathrm{eV}$ relative to vacuum, and the resulting band gap agrees very well with the experimentally measured band gap of polyethylene ${ }^{35}(8-9$ $\mathrm{eV}$ ). No adjustment was made therefore for the energies of the states in the unfilled bands (band (c) in Fig. 2 and the higher energy band not shown there). The interaction between donor (acceptor) and bridge orbitals and the composition (coefficients) of molecular orbitals are obtained directly from the extended-Hückel calculation without any adjustment.

For the sequential method, the tight-binding Hamiltonian is obtained from the same extended Hückel program, but all the interactions beyond nearest neighbors are now ignored 


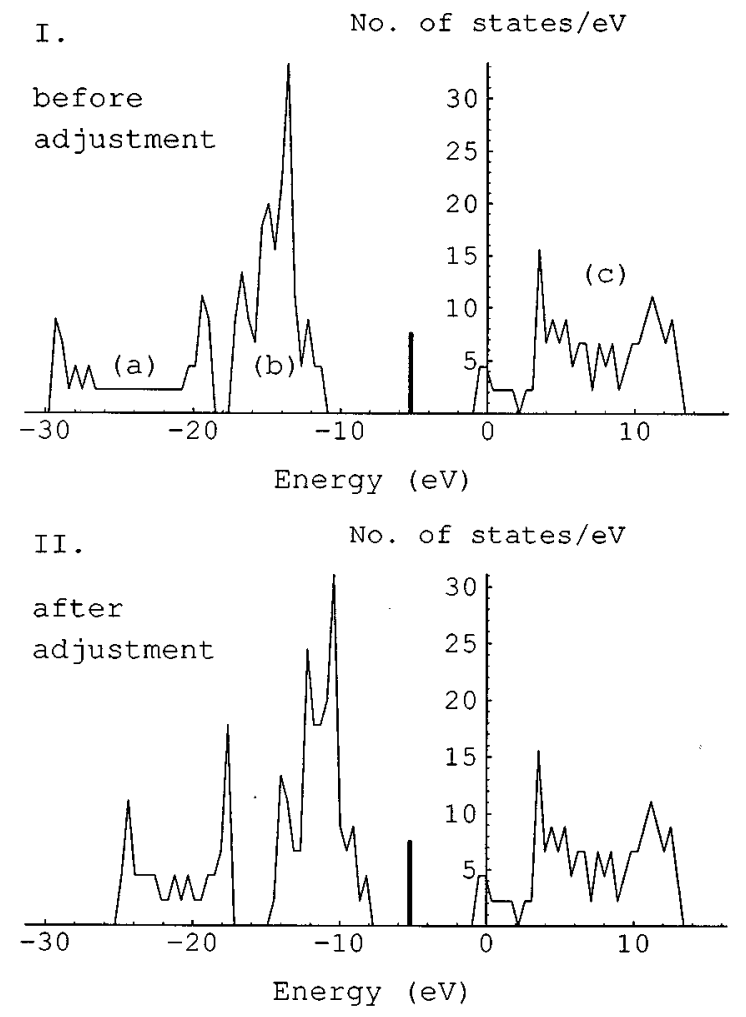

FIG. 2. The statistics of the energy levels of $\mathrm{C}_{40} \mathrm{H}_{80}$, from the extended Hückel calculation. Part I shows the statistics of the energy levels before any adjustment. Part II shows these energy levels after the adjustment described in text. The thick bar indicates the position of the energy of the electron being transferred at the transition state $(-5.22 \mathrm{eV})$. Bands (a) and (b) are valence bands. There is a band located between $26 \mathrm{eV}$ and $72 \mathrm{eV}$ that is not shown in this figure. It and band (c) are conduction bands. In the counting the energy axis was divided into cells of $0.66 \mathrm{eV}$.

and the overlap integrals are considered within each bridge unit only. The molecular orbitals of individual bridge units are obtained by solving the secular equation of each unit. Fig. 3, part I shows the distribution of energy levels from such a tight-binding Hamiltonian. It is necessary to ensure that both the upper edge of the valence band (the HOMO) and the lower edge of the conduction band (the LUMO) of the bridge agree with the experimental values. From the observed band gap $(8-9 \mathrm{eV})^{35}$ and the ionization potential $(8.8$ $\mathrm{eV})^{34}$ of the bridge, these values are $-8.8 \mathrm{eV}$ and -0.8 to $0.2 \mathrm{eV}$, respectively. The calculated band gap is only $6.2 \mathrm{eV}$ in Fig. 3, part I, which is smaller than experimental values. To obtain a better agreement with the band gap measurement, some of the six molecular orbital energies of a $\mathrm{CH}_{2}$ unit was adjusted. The third and fourth states (the $\mathrm{CH}_{2}$ HOMO and LUMO, respectively) were found to have a large effect on the states close to the band edges, those two energies were shifted by $-1 \mathrm{eV}$ and $+1 \mathrm{eV}$, respectively, an adjustment which served to give a larger band gap $(7.4 \mathrm{eV})$. All of the six MO energies were then shifted upward by 2.0 $\mathrm{eV}$ so that the upper band edge of the highest filled band agrees with the ionization potential of polyethylene. (This shift has no effect on the band gap.) With these corrections the HOMO of the bridge is $-8.8 \mathrm{eV}$ and the LUMO is -1.4

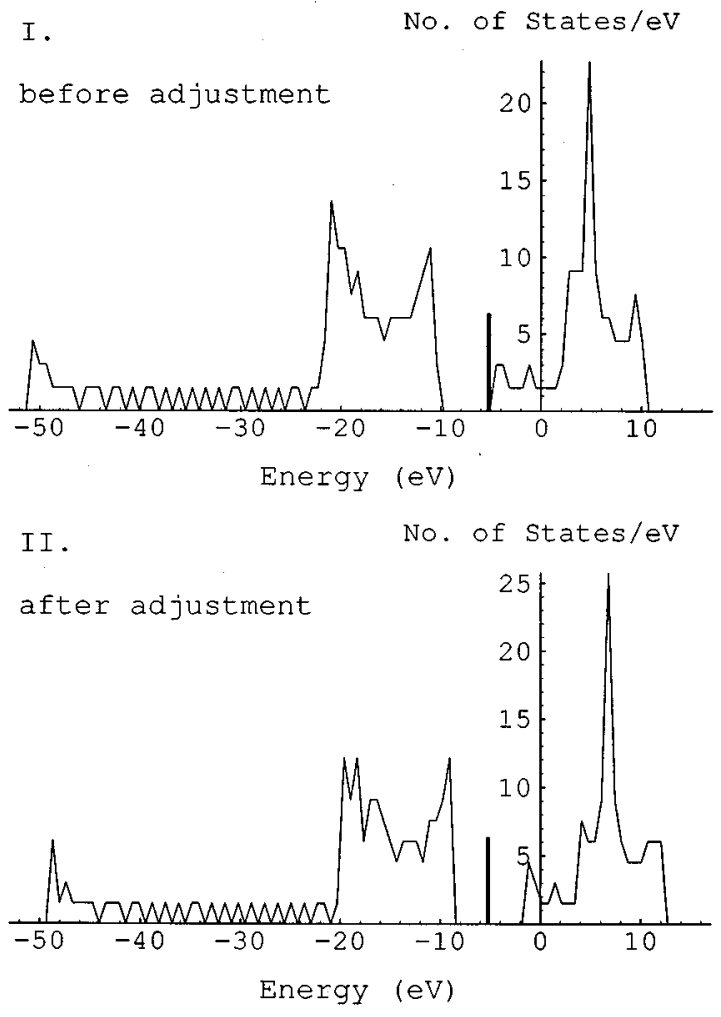

FIG. 3. Similar to Fig. 2. Part I shows the statistics of the energy levels of $\mathrm{C}_{40} \mathrm{H}_{80}$ from the tight-binding Hamiltonian, while part II shows the statistics of the energy levels after the adjustment described in text. From $30 \mathrm{eV}$ to $35 \mathrm{eV}$, there is another unfilled band which is not shown. The cell size is now $0.45 \mathrm{eV}$.

$\mathrm{eV}$, which are moderately close to the above experimental values. The MO energies after the above adjustments are now used as the diagonal matrix elements in Eq. (9). The interaction matrix elements between nearest neighbors, denoted by $\mathbf{v}$ in Section II, are obtained from the extendedHückel calculation using the MO's of each bridge unit as the basis. The distribution of energy levels from the adjusted Hamiltonian is plotted in Fig. 3, part II.

The trend of coupling strength with length of chain calculated from the sequential method is shown in Fig. 4. The

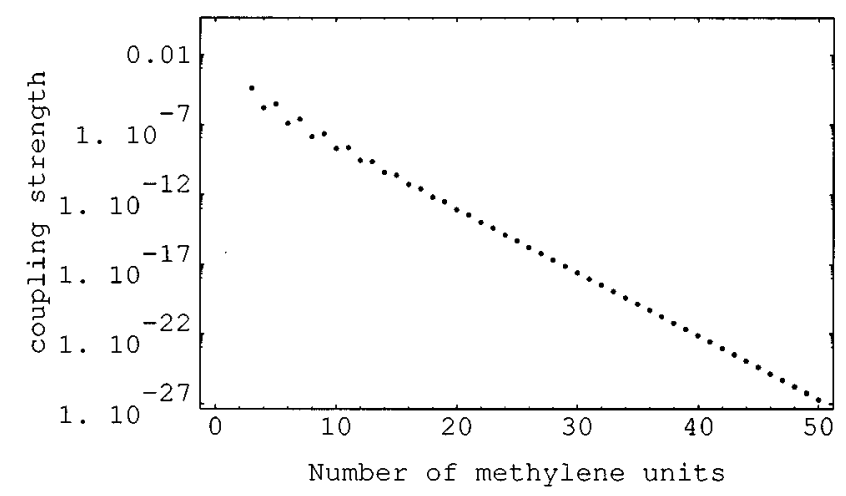

FIG. 4. Semi-log plot for $\overline{\left|H_{D k}\right|^{2}}$ as a function of number of methylene units in the bridge. Results are obtained from the sequential formula. 


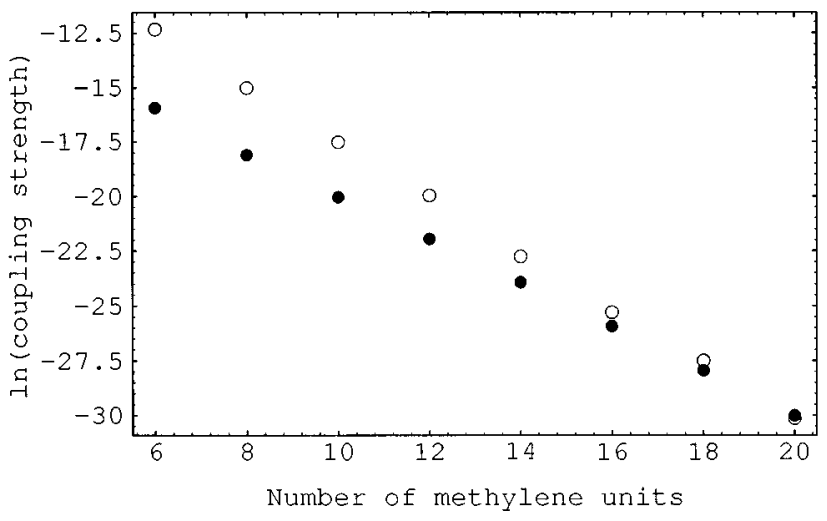

FIG. 5. A plot for $\log _{e} \overline{\left|H_{D k}\right|^{2}} v$ s. different bridge lengths. " $\bigcirc$ " denotes that for direct summation, "@" from the sequential formula. The difference between the two sets of data is mainly due to their Hamiltonians. See text for the discussions.

results of both the direct summation and sequential methods are given in Fig. 5. Various aspects of there results are discussed and compared next.

We have also calculated the effect of additional parallel alkane thiol chains using the structure from Klein. ${ }^{25}$ For one of the possible conformations of the ferrocenylcarboxyl group ${ }^{28}$ the effective coupling calculated from direct summation method for different numbers of additional chains is listed in Table I.

\section{DISCUSSION}

In the following discussion, we first consider and compare the results shown in Figs. 4 and 5 and in Table I. We then discuss the generalization to a more complicated bridge system that has different bridge units or that has a complex geometrical structure instead of being a linear chain. The flexibility of the graph-based method described in the Appendix will be discussed in the end of that section.

Using Eqs. (30), (31), the sequential formula (Eqs. (22) and (23)), Eq. (33) and the experimental value of $\lambda,{ }^{7} 0.85$ $\mathrm{eV}$, we obtained a coupling strength $\left(\overline{\left|H_{D k}\right|^{2}}\right)$ of about $5.6 \times 10^{-12} \mathrm{eV}^{2}$ atom for $n=16$, which yields a rate constant of about $0.11 \mathrm{sec}^{-1}$. In obtaining the latter, the density of states of gold electrode was estimated from the tight-binding formalism by a Monte Carlo method. At the Fermi energy of gold, the value $0.05 \pm 0.002 / \mathrm{eV} /$ atom was obtained. As a comparison, if the density of states of Au at the Fermi level, obtained from low temperature specific heat, 0.3/eV/atom

TABLE I. The effective coupling strengths for $n=12$, with additional thiol molecules.

\begin{tabular}{cc}
\hline \hline $\begin{array}{c}\text { No. of additional } \\
\text { thiol chains }\end{array}$ & $\frac{\text { effective coupling }}{\left|H_{D k}\right|^{2}} \times 10^{9}, \mathrm{eV}^{2}$ atom \\
\hline 0 & 2.15 \\
1 & 1.88 \\
2 & 1.95 \\
3 & 1.94 \\
\hline \hline
\end{tabular}

(Ref. 37) is used instead, one would obtain a reaction rate that is a factor of 6 larger. The reaction rates calculated above roughly agree, within one order of magnitude, with the $1.25 \mathrm{sec}^{-1}$ measured from the electrochemical exchange current voltage by Chidsey. ${ }^{7}$

The sequential method is numerically easy to calculate with good precision. In Fig. 4 the coupling strength calculated by the sequential method is shown for up to 50 bridge units $\left(-\mathrm{CH}_{2}-\right){ }^{36}$ It can be seen that for longer bridge chains, there is an excellent exponential decay, while for shorter chains, the decay is modulated with an initial oscillation. Fitting the electronic coupling of the long chains $(n=30-50)$ with a term proportional to $\exp (-\beta n)$ yields $\beta=1.05$ per methylene unit. For even-numbered short chains $(n=6-20)$ the value calculated for $\beta$ is 1.00 per methylene unit.

The direct summation method gives an electronic coupling strength for short chains similar to that for the sequential method. The linear fit for the result of the direct summation yields $\beta=1.27$ per methylene unit for $n=6-20$. These data decay with $n$ slightly differently from that of sequential method because the Hamiltonian for both cases is not exactly the same. For the present form of the sequential method we need to use a tight-binding approximation which neglects all the interaction beyond nearest neighbor units. For the direct summation calculation, on the other hand, all Hamiltonian matrix elements generated by the extended-Hückel program were included. It should be stressed, however, that since the sequential formula for the bridge Green's function (Eqs. (22) and (23)) is mathematically exact and numerically stable, the difference in the two sets of data points in Fig. 5 arises only from the difference in the two model Hamiltonians. The Hamiltonians have been adjusted independently, as described in Section III C, to agree better with the experimental band structure measurements.

We also calculated the coupling strength through hole transfer mechanism by doing the direct summation only over the filled states of bridge part. Our result shows that the hole transfer scheme provides the major pathways of the coupling, and it yields more than $89 \%$ of the total coupling strength.

For a long chain bridge, direct summation method performs summations (instead of merely multiplications) resulting in a large amount of cancellation to yield a small value. So numerically it requires more care for the number of significant figures of interaction matrix elements as well as the coefficients in describing MO's with atomic orbitals, and the method can be expected to fail when the effective coupling strength is smaller than the significant digits of the numbers being summed. The direct summation calculation, even after all the bridge states were pre-diagonalized, also still required at least ten times more computer time than that for the sequential method.

Our sequential calculation is based on a tight-binding Hamiltonian. Matrix elements and overlap integrals from the extended-Hückel program are not always the best choice for the tight-binding model. In our calculation we found that the band gap obtained in this tight-binding model is too small (it 


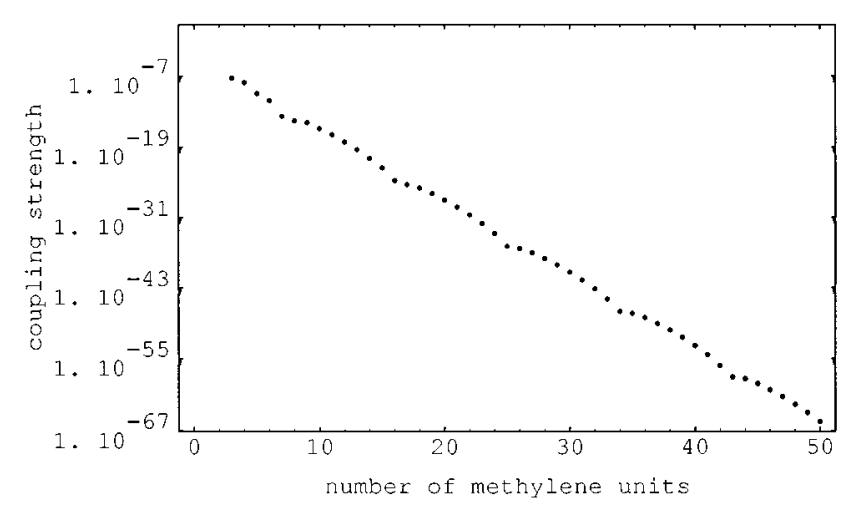

FIG. 6. Semi-log plot for the same calculation as in Fig. 4, except the energy of the electron is shifted to another region for the purpose of demonstrating the effect of oscillation caused by the pair of conjugate complex eigenvalues of the matrix $\mathbf{v} \Delta^{-1} N_{n}$.

is about $6.2 \mathrm{eV}$ ) compared with that from experiment (8-9 eV Ref. 35). The band structure of the tight-binding model also does not resemble that from full extended-Hückel calculation. Since the energy of the electron being transferred lies between the conduction band and the valence band of polyethylene, the position of the band edges are a most crucial factor in determining the effective coupling across the hydrocarbon chain. In the sequential (nearest-neighbor tightbinding) calculation we adjusted the position of two of the MO energies of $\mathrm{CH}_{2}$, as described in Section III, so that the band gap is larger than the $6.2 \mathrm{eV}$, namely $7.4 \mathrm{eV}$, which is fairly close to that of experiments. In applying this method to other systems, the tight-binding Hamiltonian for the bridge part used should fit band structure measurements.

Turning now to the effect studied in Table I, there is seen to be little effect from additional chains. This result indicates that in this system electron transfer occurs mainly through the chain covalently bonded to the redox active group. The closest atom-to-atom distance from the first added alkane thiol molecule to the ferrocenylcarboxyl group is $1.6 \AA .{ }^{28}$ Even with such close contact between molecules, the additional thiol chains still do not effectively provide an alternate route for the electron to be transferred to the electrode, according to the results in Table I.

In Fig. 6 is a test of the condition discussed at the end of Section II, where the energy of electron is deliberately shifted to a place where the matrices $\left\{\mathbf{v} \Delta^{-1} N_{n}\right\}$ are almost constant with respect to the iteration (Eq. (22)), but where two of the important eigenvalues are a pair of conjugate complex numbers. For this alkane bridge chain the energy needed for this effect to occur is not physically accessible, but it is still possible for other homogeneous chain bridges to have this kind of oscillatory length dependence. Moreover, there is no corresponding trend in a single-band model: in an off-resonance, single-band system, $\left\{\mathbf{v} \Delta^{-1} N_{n}\right\}$ is a scalar series, and therefore the magnitude of $G_{(1, n)}^{(n)}$ (which is also a scalar now) decays monotonically as $\mathbf{v} \Delta^{-1} N_{n}$ goes to a small constant real or complex number.

As mentioned earlier, in the derivation in Section II it is not required to have a bridge of identical subunits. For dif- ferent bridge units with either the same or a different number of orbitals, $\mathbf{v}$ is now a square or rectangular matrix, respectively, describing the interaction between specific neighboring bridge sites, $n$ and $(n-1)$ in Eqs. (22) and (23), and the diagonal matrix $\Delta$ must be then labeled with a subscript $n$, e.g., $\Delta_{n}$. Eqs. (22) and (23) now become

$$
\begin{aligned}
& N_{n}=\left(\mathbf{1}-\mathbf{v}_{(n-1, n)}^{T} \Delta_{n-1}^{1} N_{n-1} \mathbf{v}_{(n-1, n)} \Delta_{n}^{-1}\right)^{-1}, \\
& G_{(1, n)}^{(n)}=G_{(1, n-1)}^{(n-1)} \mathbf{v}_{(n-1, n)} \Delta_{n}^{-1} N_{n},
\end{aligned}
$$

where $\mathbf{v}_{(n-1, n)}$ denotes the interaction matrix between the $(n-1)$ th and the $n$th bridge units. The initial condition $G_{1,2}^{(2)}$ is defined as the corresponding matrix between the first and the second bridge units. In this way, the Green's function can be obtained for an arbitrary tight-binding linear chain bridge, without solving a large linear system. There are already strategies for solving such a large set of linear equations, e.g., that of Stuchebrukhov, ${ }^{5}$ and others, ${ }^{11}$ and it will be interesting to compare those methods with the present sequential method, both with respect to computation and physical insight.

Formally in applying the sequential formula the only limit on the range of energy of transferred electron is that it should not be coincident with the poles of $\Delta^{-1}$ and of the final Green's function $G^{(n)}$. However, the Green's function for the bridge is not a good approximation for the overall Green's function when the energy is close to one of the energy levels of the bridge. Also, the physical situation of an on-resonant system is quite different. It may not even involve an electron transfer from a state localized on the donor to one localized on the acceptor.

A further generalization can be made for more complicated structures of bridges using the graph-based method described in the Appendix.

\section{CONCLUSION}

The sequential formula (Eqs. (22) and (23)) developed in the present paper is numerically stable even for the case of a large number of bridge orbitals. Since it involves only the inversion and multiplication of small matrices whose sizes are independent of the chain length, it is also much less computationally time-consuming than the direct summation method.

This new method can be applied to various kinds of bridge molecules and, we believe, by extending the oneorbital per site case to many orbitals per site, as in the present paper, provides added physical insight into various effects. To the best of our knowledge, the present work appears to be the first that rigorously treats a multiple band, tight-binding Hamiltonian and, thereby, the origin of and the condition for the exponential dependence for such cases. As seen in the calculation, by investigating conditions for constancy of $N_{n}$ in the case of a uniform bridge, it directly reflects the origin of any exponential or other regular dependence (Fig. 6) for multiband systems. This exponential dependence for an off-resonant bridges was assumed, and reasonably so, in earlier work. ${ }^{4}$ For a linear bridge, no further 
assumption need be made for the calculation of the coupling strength, apart from the tight-binding Hamiltonian and, in the present case, using the Green's function for bridge subspace instead of that of the whole donor-bridge-acceptor system. The method can be applied to a wide class of systems, including non-uniform bridges and could be extended to nonlinear bridge molecules, perturbatively if necessary.

\section{ACKNOWLEDGMENTS}

It is a pleasure to acknowledge the support of the National Science Foundation, the Office of Naval Research, and NEDO (Japan), and the very helpful comments of Mark Ratner and Aseem Mehta.

\section{APPENDIX}

\section{A graph-based approach}

Another approach of deriving the sequential formula, using a graph representation for the terms in the expansion of the Green's function, is given in this Appendix. This method yields the same final answer as that derived in Section II (Eqs. (22) and (23)), and it provides a physical picture of the coupling scheme. Thereby, this graph-based method may prove useful in generalizing the calculation for more complex structures of the bridge.

\section{The Hamiltonian and the Green's function}

Using Eq. (8), instead of treating only the interaction between the last two bridge units as a perturbation $\left(\mathscr{H}_{1}\right)$, we now regard all the off-diagonal matrix elements as perturbations $\left(H^{\prime}\right)$ and so $H_{0}$ now contains only diagonal matrix elements. For simplicity of presentation, the case of identical bridge units will be considered. The new zeroth order Hamiltonian is, thereby,

$$
H_{0}=\left(\begin{array}{cccccc}
\mathbf{e} & \mathbf{0} & \mathbf{0} & \mathbf{0} & \cdots & \mathbf{0} \\
\mathbf{0} & \mathbf{e} & \mathbf{0} & \mathbf{0} & \cdots & \vdots \\
\mathbf{0} & \mathbf{0} & \mathbf{e} & \mathbf{0} & \cdots & \vdots \\
\vdots & \vdots & \vdots & \vdots & \ddots & \mathbf{0} \\
\mathbf{0} & \cdots & \cdots & \mathbf{0} & \mathbf{0} & \mathbf{e}
\end{array}\right),
$$

and the perturbation $H^{\prime}$ is now

$$
H^{\prime}=\left(\begin{array}{cccccc}
\mathbf{0} & \mathbf{v} & \mathbf{0} & \mathbf{0} & \cdots & \mathbf{0} \\
\mathbf{v}^{T} & \mathbf{0} & \mathbf{v} & \mathbf{0} & \cdots & \vdots \\
\mathbf{0} & \mathbf{v}^{T} & \mathbf{0} & \mathbf{v} & \cdots & \vdots \\
\vdots & \vdots & \vdots & \vdots & \ddots & \mathbf{v} \\
\mathbf{0} & \cdots & \cdots & \mathbf{0} & \mathbf{v}^{T} & \mathbf{0}
\end{array}\right),
$$

where the block matrices $\mathbf{e}, \mathbf{v}$ and $\mathbf{0}$ are the same as defined in Section II. Thus, the Green's function can be expressed as the following expansion:

$$
\begin{aligned}
G & =\left(E \mathbf{1}-H_{0}-H^{\prime}\right)^{-1} \\
& =G_{0}+G_{0} H^{\prime} G_{0}+G_{0} H^{\prime} G_{0} H^{\prime} G_{0}+\cdots,
\end{aligned}
$$

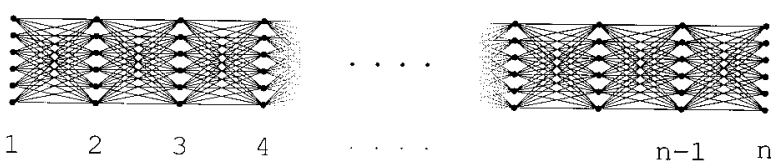

FIG. 7. Graph representation for the multiple-band tight-binding Hamiltonian. Each vertical column represents a bridge unit. The dots in each column correspond to molecular orbitals in each bridge unit. Lines connecting two dots represent the coupling between the two orbitals, represented by the pair of dots. The lines only connect dots in nearest-neighbor columns. To evaluate the Green's function, all possible pathways from one dot to the other are included, as described in the text.

where $G_{0}$, the Green's function corresponding to $H_{0}$, is now the inverse of a diagonal matrix

$$
G_{0}=\left(E \mathbf{1}-H_{0}\right)^{-1},
$$

or,

$$
\left(G_{0}\right)_{n_{i}, m_{i} ; n_{j}, m_{j}}=\delta_{n_{i}, n_{j}} \delta_{m_{i}, m_{j}} \frac{1}{E-\varepsilon_{m_{i}}},
$$

where $n_{i}$ is the index for the $n_{i}$ th bridge unit, so the indices $n_{i}$ and $n_{j}$ refer to the block matrix at $\left(n_{i}, n_{j}\right)$ position of $G_{0}$. Similarly, $m_{i}$ is the index for the $m_{i}$ th molecular orbital, therefore $m_{i}$ and $m_{j}$ refer to a matrix element inside the $\left(n_{i}, n_{j}\right)$ block.

\section{Graph representation}

One way of calculating $G$ in Eq. (A3) is to draw a graph whose vertices represent zeroth-order states and where lines exist between two states only if they interact (Fig. 7). We then make use of a theorem which states that there is a oneto-one correspondence between each possible path on such a graph and each term in each of the matrix element products in the complete expansion of the Green's function. ${ }^{14}$ The connection is as follows: each path begins and ends with a dot, between two dots the path is connected with a line. The corresponding quantity obtained from the path is a product of the following factors:

$$
\begin{aligned}
& \text { for every dot it visits: } 1 /\left(E-\varepsilon_{m_{i}}\right) \text {; } \\
& \text { for every line: } \mathbf{v}_{m_{i}, m_{j}} .
\end{aligned}
$$

For each overall matrix element there are an infinite number of paths corresponding to it, but by classifying the paths in a suitable way, it is often possible to obtain the exact expression. One example is the RPE (renormalized perturbative expansion) which selects the "skeleton" (self-avoiding) paths and then adds "decoration" to make an arbitrary path. ${ }^{14,38}$

We consider the part of $G$ that is needed to obtain the effective coupling strength, and denote it now by $G_{(1, n)}^{(n)}\left(m_{1}, m_{n}\right)$. It consists of all possible paths from any orbital $m_{1}$ in the first site to any orbital $m_{n}$ in the last $(n$ th) site. At the vertical column of points for the $(n-1)$ th site (Fig. 8) all such paths must cross this column at least once and all of them cross it an odd number of times. 

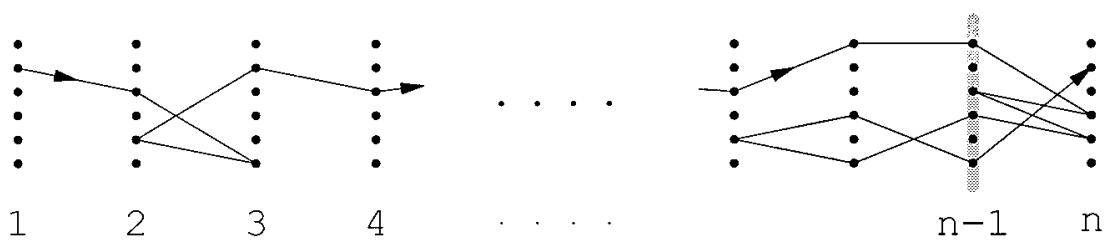

FIG. 8. A possible path that crosses the dividing column (shaded bar at site $n-1$ ) three times. ("Visiting'" but then returning does not count as "crossing.")

One can then cut the paths into pieces at the place where they cross this dividing line. The paths are composed of segments that are either within the first $n-1$ units or wandering between the $(n-1)$ th and the $n$th units. The former type of segments are related to the Green's function for $(n-1)$ bridge units, $G^{(n-1)}$, while the latter type of segment is a computable quantity.

We define the segments as the paths without their beginning dots so that there is no confusion upon connecting segments into a longer piece. The corresponding terms with respect to such segments, i.e., matrix elements apart from the initial $1 /(E-\varepsilon)$ factor (Eq. (A6)), is denoted as $F$, with proper superscript and notation defined later. $F$ will represent this new set of matrices modified from Green's function $G$. In order to describe all possible variations of different molecular orbitals in the same unit, our notation is for $m \times m$ matrices, with specific indices for the starting and ending sites only; the matrix elements of these $m \times m$ matrices correspond to orbital to orbital transition. So described, we define $F_{(1, n)}^{(n)}$ by:

$$
G_{(1, n)}^{(n)}=\Delta^{-1} F_{(1, n)}^{(n)},
$$

where $\Delta$ has already been defined in Eq. (14). The inverse $\Delta^{-1}$ is the matrix corresponding to the dots that each path starts with, and $G_{(1, n)}^{(n)}$ has the same definition as in Section II. We have, thereby,

$$
F_{(1, n)}^{(n)}=\sum \quad(\text { all possible segments from unit } 1 \text { to } n) .
$$

As discussed above, all of segments corresponding to $F_{(1, n)}^{(n)}$ cross the dividing line (the shaded bar in Fig. 8) an odd number of times. So the segments can be classified by the number of crossings, i.e.,

$F_{(1, n)}^{(n)}=\sum$ (segments that cross the $n-1$ site once)

$+\sum$ (segments that cross three times)

$+\cdots+\sum$ (segments that cross $2 j+1$ times $)+\cdots$.

(A10)

In the following sections, expressions for terms in Eq. (A10) are derived.

\section{The first term in calculating $F_{(1, n)}^{(n)}$}

The first term representing segments crossing site $n-1$ once is given by:

$\sum($ segments that cross once $)=F_{(1, n-1)}^{(n-1)} F_{(n-1, n)}^{(2)}$,

which is a sum over of all possible segments from site 1 to site $(n-1)$ multiplied by the sum over all possible segments that go from site $(n-1)$ to site $n$. Since a uniform bridge is treated in the present argument, the transition from bridge site $(n-1)$ to site $n$ is the same as that for any other two neighboring sites. In Eq. (A11) such a transition is denoted by $F_{(n-1, n)}^{(2)}$. This quantity can be obtained by classifying all the segments as follows:

(1) The segments that go directly from site $n-1$ to site $n$, in only 1 step: those segments contain only one line and one dot on site $n$, so the corresponding terms are the appropriate elements of $\mathbf{v}$ times the appropriate elements of $\Delta^{-1}$ according to Eqs. (A6) and (A7). Thereby, this contribution to $F_{(n-1, n)}^{(2)}$ is $\mathbf{v} \Delta^{-1}$, which accounts for all 1-step segments from any orbital in site $n-1$ to any orbital in site $n$.

(2) The segments that bounce back and forth between site $n-1$ and site $n$ : An example of such a segment is shown in Fig. 9. By an argument similar to the one above we obtain $\mathbf{v} \Delta^{-1} \mathbf{v}^{T} \Delta^{-1} \mathbf{v} \Delta^{-1}$ for segments that return to site $n-1$ once and end up at site $n$, and $\left(\mathbf{v} \Delta^{-1} \mathbf{v}^{T} \Delta^{-1}\right)^{2} \mathbf{v} \Delta^{-1}$ for segments that return twice, etc.

In this way $F_{(n-1, n)}^{(2)}$ is obtained by a summation over the above two contributions:
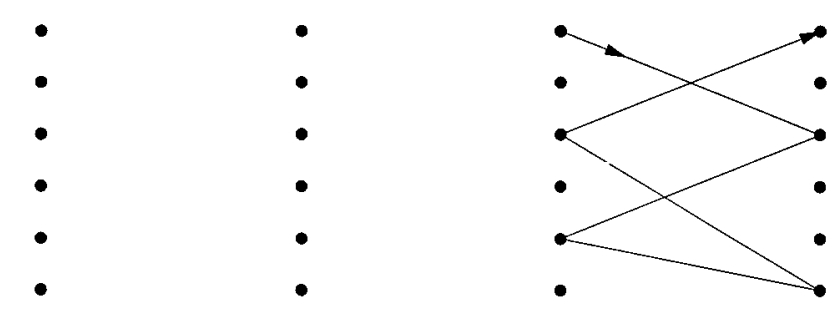

$n-2 \quad n-1$

n

FIG. 9. A possible path for calculating $F_{(n-1, n)}^{(2)}$. 


$$
\begin{aligned}
F_{(n-1, n)}^{(2)}= & \mathbf{v} \Delta^{-1}+\mathbf{v} \Delta^{-1} \mathbf{v}^{T} \Delta^{-1} \mathbf{v} \Delta^{-1} \\
& +\mathbf{v} \Delta^{-1} \mathbf{v}^{T} \Delta^{-1} \mathbf{v} \Delta^{-1} \mathbf{v}^{T} \Delta^{-1} \mathbf{v} \Delta^{-1}+\cdots \\
= & \left(1-\mathbf{v} \Delta^{-1} \mathbf{v}^{T} \Delta^{-1}\right)^{-1} \mathbf{v} \Delta^{-1}
\end{aligned}
$$

This particular matrix also serves as the initial matrix for $F_{(1, n)}^{(n)}$ for a uniform bridge. For non-uniform bridges, the initial condition for $F_{(1, n)}^{(n)}$ needs to be calculated for the first two units, using the same expression as in Eq. (A12), but with appropriate matrices $\mathbf{v}$ and $\Delta$.

\section{The second and other terms in calculating $F_{(1, n)}^{(n)}$}

In Section $\mathrm{C}$ of the Appendix, it is seen that for segments contributing $F_{(1, n)}^{(n)}$ the number of times they cross the ( $n-1)$ dividing line (Fig. 8) should be an odd number (Eq. (A10)). All the possible segments that cross the dividing line three times are next considered. These segments must be composed of 4 segments: The first segment goes from site 1 to site $n-1$, and its corresponding matrix has already been defined as $F_{(1, n-1)}^{(n-1)}$. The second segment starts from the same final orbital at site $n-1$, and it goes to site $n$ and then returns to site $n-1$ including bouncing between the two sites for any number of times. We denote this part of the contribution by $F_{(n-1, n-1)}^{(2)}$. It differs from $F_{(n-1, n)}^{(2)}$ in that it finally returns to site $n-1$. The third segment starts from the $n-1$ site, visits the space of the first $n-1$ sites, where it involves an arbitrary "loop" within the first $n-1$ sites, and, it then returns to the $n-1$ site. We denote the matrix for such segments by $F_{(n-1, n-1)}^{(n-1)}$ The final segment goes from any orbital in site $n-1$ to any orbital of site $n$ arbitrarily, so it corresponds to a matrix element of $F_{(n-1, n)}^{(2)}$ as shown in Section D of the Appendix. Thereby, we have

$\sum$ (segments that cross the $n-1$ site 3 times)

$$
=F_{(1, n-1)}^{(n-1)} F_{(n-1, n-1)}^{(2)} F_{(n-1, n-1)}^{(n-1)} F_{(n-1, n)}^{(2)} .
$$

Generalizing the above expression to any odd number of crossings of the $n-1$ sites gives

$\sum$ (segments that cross $2 j+1$ times)

$$
=F_{(1, n-1)}^{(n-1)}\left[F_{(n-1, n-1)}^{(2)} F_{(n-1, n-1)}^{(n-1)}\right]^{j} F_{(n-1, n)}^{(2)} .
$$

To evaluate Eq. (A10), a summation of the terms in Eq. (A14) for $j=0$ to $\infty$ yields

$$
\begin{aligned}
F_{(1, n)}^{(n)}= & F_{(1, n-1)}^{(n-1)}\left[\mathbf{1}+F_{(n-1, n-1)}^{(2)} F_{(n-1, n-1)}^{(n-1)}\right. \\
& \left.+\left(F_{(n-1, n-1)}^{(2)} F_{(n-1, n-1)}^{(n-1)}\right)^{2}+\cdots\right] F_{(n-1, n)}^{(2)} \\
= & F_{(1, n-1)}^{(n-1)}\left[\mathbf{1}-F_{(n-1, n-1)}^{(2)} F_{(n-1, n-1)}^{(n-1)}\right]^{-1} F_{(n-1, n)}^{(2)}, \\
& \quad \text { for } n=3,4,5, \cdots .
\end{aligned}
$$

Also, Eq. (A15) can be rewritten in terms of $G_{(1, n)}^{(n)}$ $G_{(1, n)}^{(n)}=G_{(1, n-1)}^{(n-1)}\left[\mathbf{1}-F_{(n-1, n-1)}^{(2)} F_{(n-1, n-1)}^{(n-1)}\right]^{-1} F_{(n-1, n)}^{(2)}$,

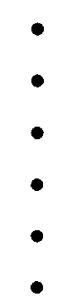

FIG. 10. A possible path for calculating $F_{(n-1, n-1)}^{(2)}$.

by using Eq. (A8) for both $G_{(1, n)}^{(n)}$ and $G_{(1, n-1)}^{(n-1)}$

So now $F_{(1, n)}^{(n)}$ is written in terms of $F_{(1, n-1)}^{(n-1)} F_{(n-1, n)}^{(2)}$ $F_{(n-1, n-1)}^{(2)}$ and $F_{(n-1, n-1)}^{(n-1)}$. The first one is the recursive variable, and the second one, $F_{(n-1, n)}^{(2)}$ has been obtained in Section D of the Appendix (Eq. (A12)). The next section is then devoted to deriving expressions for $F_{(n-1, n-1)}^{(2)}$ and $F_{(n-1, n-1)}^{(n-1)}$

\section{The expressions for $F_{(n-1, n-1)}^{(2)}$ and $F_{(n-1, n-1)}^{(n-1)}$}

We next evaluate $F_{(n-1, n-1)}^{(2)}$ which is the matrix for the sum of the terms corresponding to all possible segments bouncing back and forth between two rows of states that start and end at the $(n-1)$ th row. In Fig. 10 is shown one of such paths. It differs from the $F_{(n-1, n)}^{(2)}$ by having a return from site $n$ to site $n-1$, contributing a matrix $\mathbf{v}^{T} \Delta^{-1}$. Thereby,

$$
\begin{aligned}
F_{(n-1, n-1)}^{(2)} & =F_{(n-1, n)}^{(2)} \mathbf{v}^{T} \Delta^{-1} \\
& =\left(\mathbf{1}-\mathbf{v} \Delta^{-1} \mathbf{v}^{T} \Delta^{-1}\right)^{-1} \mathbf{v} \Delta^{-1} \mathbf{v}^{T} \Delta^{-1} .
\end{aligned}
$$

From the above definition and discussion, $F_{(n-1, n-1)}^{(n-1)}$ is related to the $(n-1, n-1)$ block of $G$ matrix in the following way:

$$
G_{(n-1, n-1)}^{(n-1)}=\Delta^{-1}\left(\mathbf{1}+F_{(n-1, n-1)}^{(n-1)}\right)
$$

in which an $m \times m$ unit matrix $\mathbf{1}$ is needed because the diagonal matrix elements of $G_{(n, n)}^{(n)}$ include "null paths",(i.e., paths which do nothing) arising from the first term $\left(G_{0}\right)$ in the expansion of Eq. (A3), and, $\Delta^{-1}$ represents the missing beginning dots for segments in calculating $F^{(n)}$ (Eq. (A6)).

By a strategy similar to that which led to $F_{(1, n)}^{(n)}$ one can derive

$$
\begin{aligned}
F_{(n, n)}^{(n)}= & \mathbf{v}^{T} \Delta^{-1}\left(\mathbf{1}+F_{(n-1, n-1)}^{(n-1)}\right) \\
& \times\left[\mathbf{1}-F_{(n-1, n-1)}^{(2)} F_{(n-1, n-1)}^{(n-1)}\right]^{-1} F_{(n-1, n)}^{(2)},
\end{aligned}
$$

in which all the possible segments for $F_{(n, n)}^{(n)}$ are divided into several parts depending on the number of times they cross the dividing site $n-1$. Those segments are classified as follows:

(1) The first segment is defined as a single step going from site $n$ directly back to site $n-1$. This segment is introduced to force $F_{(n, n)}^{(n)}$ to contain no null segment, and it corresponds to $\mathbf{v}^{T} \Delta^{-1}$. 
(2) The second segment is either a null one (1) or any loop that begins and stops at $(n-1)$ th site $\left(F_{(n-1, n-1)}^{(n-1)}\right)$.

(3) On the other hand, the final segment is composed of any number of steps that go from the dividing line (site $n-1)$ to a final stop on site $n$. This part exactly corresponds to the definition of $F_{(n-1, n)}^{(2)}$.

(4) There can be any number of segments between the second one and the final one. Those segments form loops in either spaces for the last two sites $\left(F_{(n-1, n-1)}^{(2)}\right)$, or the first $(n-1)$ sites $\left(F_{(n-1, n-1)}^{(n-1)}\right)$. This set of segments includes the null path so the first term should be $\mathbf{1}$. Two consecutive loops at the same side would contribute to the single $F_{(n-1, n-1)}^{(n-1)}$ or $F_{(n-1, n-1)}^{(2)}$ matrix because of its definition. If the final loop is in the last two sites $\left(F_{(n-1, n-1)}^{(2)}\right)$, it can also be regarded as a part of the segments corresponding to $F_{(n-1, n)}^{(2)}$ which is the final segment as describe above. To avoid over-counting, it is then required to have the last loop at the side of the first $(n-1)$ site $\left(F_{(n-1, n-1)}^{(n-1)}\right)$. So the contribution of this part of segment is:

$$
\begin{aligned}
\mathbf{1}+ & F_{(n-1, n-1)}^{(2)} F_{(n-1, n-1)}^{(n-1)} \\
& +F_{(n-1, n-1)}^{(2)} F_{(n-1, n-1)}^{(n-1)} F_{(n-1, n-1)}^{(2)} F_{(n-1, n-1)}^{(n-1)}+\cdots \\
= & {\left[\mathbf{1}-F_{(n-1, n-1)}^{(2)} F_{(n-1, n-1)}^{(n-1)}\right]^{-1} . }
\end{aligned}
$$

By multiplying the above four factors together, Eq. (A19) for $F_{(n, n)}^{(n)}$ was obtained.

\section{The sequential formula}

Eq. (A16) is the sequential formula derived in this appendix. To show the equivalence between Eq. (19) in the text and this Eq. (A16), the following identity can be derived:

$$
\begin{aligned}
& {\left[\mathbf{1}-F_{(n-1, n-1)}^{(2)} F_{(n-1, n-1)}^{(n-1)}\right]^{-1} F_{(n-1, n)}^{(2)} } \\
&=\left[\mathbf{1}-\mathbf{v} \Delta^{-1}\left(\mathbf{1}+\mathbf{v}^{T} \Delta^{-1} \mathbf{v} \Delta^{-1}+\cdots\right) \mathbf{v}^{T}\right. \\
&\left.\times \Delta^{-1} F_{(n-1, n-1)}^{(n-1)}\right]^{-1} \times \mathbf{v} \Delta^{-1}\left(\mathbf{1}+\mathbf{v}^{T} \Delta^{-1} \mathbf{v} \Delta^{-1}\right. \\
&\left.+\mathbf{v}^{T} \Delta^{-1} \mathbf{v} \Delta^{-1} \mathbf{v}^{T} \Delta^{-1} \mathbf{v} \Delta^{-1}+\cdots\right) \\
&= \mathbf{v} \Delta^{-1}\left[\mathbf{1}-\left(\mathbf{1}-\mathbf{v}^{T} \Delta^{-1} \mathbf{v} \Delta^{-1}\right)^{-1} \mathbf{v}^{T}\right. \\
&\left.\times \Delta^{-1} F_{(n-1, n-1)}^{(n-1)} \mathbf{v} \Delta^{-1}\right]^{-1}\left(\mathbf{1}-\mathbf{v}^{T} \Delta^{-1} \mathbf{v} \Delta^{-1}\right)^{-1} \\
&= \mathbf{v} \Delta^{-1}\left[\mathbf{1}-\mathbf{v}^{T} \Delta^{-1}\left(\mathbf{1}+F_{(n-1, n-1)}^{(n-1)}\right) \mathbf{v} \Delta^{-1}\right]^{-1},
\end{aligned}
$$

in which Eqs. (A12) and (A17) were used. Therefore, with Eq. (A18)

$$
\begin{aligned}
{[\mathbf{1}-} & \left.F_{(n-1, n-1)}^{(2)} F_{(n-1, n-1)}^{(n-1)}\right]^{-1} F_{(n-1, n)}^{(2)} \\
& =\mathbf{v} \Delta^{-1}\left(\mathbf{1}-\mathbf{v}^{T} G_{(n-1, n-1)}^{(n-1)} \mathbf{v} \Delta^{-1}\right)^{-1} .
\end{aligned}
$$

So Eq. (A16) becomes Eq. (19) with the above identity (Eq. (A22)) introduced.

Also, if the expression Eq. (A21) is introduced into Eq. (A19), the following identity is obtained:

$$
\begin{aligned}
\mathbf{1}+F_{(n, n)}^{(n)}= & \mathbf{1}+\mathbf{v}^{T} \Delta^{-1}\left(\mathbf{1}+F_{(n-1, n-1)}^{(n-1)}\right) \mathbf{v} \Delta^{-1} \\
& \times\left(\mathbf{1}-\mathbf{v}^{T} \Delta^{-1}\left(\mathbf{1}+F_{(n-1, n-1)}^{(n-1)}\right) \mathbf{v} \Delta^{-1}\right)^{-1} \\
= & {\left[\mathbf{1}-\mathbf{v}^{T} \Delta^{-1}\left(\mathbf{1}+F_{(n-1, n-1)}^{(n-1)}\right) \mathbf{v} \Delta^{-1}\right]^{-1} . }
\end{aligned}
$$

Together with Eq. (A18), the above expression is equivalent to Eq. (20). Comparison of Eqs. (21) and (A18) shows that

$$
N_{n}=\mathbf{1}+F_{(n, n)}^{(n)} \text {. }
$$

With this identity (Eq. (A24)), Eq. (A23) is seen to be the same as Eq. (22).

\section{Discussion}

This approach, beginning with an infinite series expansion (Eq. (A3)), gives the same recursion relation as derived in Section II by a non-perturbative method. Mathematically, the infinite series expansion in Eq. (A3) converges inside its radius of convergence, namely when the modulus of every eigenvalue of the matrix $G_{0} H^{\prime}$ (or $H^{\prime} G_{0}$ ) is less than unity. A similar restriction appears in Eqs. (A15) and (A17), which requires both $F_{(n-1, n-1)}^{(2)} F_{(n-1, n-1)}^{(n-1)}$ and $\mathbf{v} \Delta^{-1} \mathbf{v}^{T} \Delta^{-1}$ to have all of their eigenvalues within the unit circle in the complex plane. However, the resulting expressions are not limited by such conditions since they can also be derived from the nonperturbative method. This aspect demonstrates a desired property of the graph-based method, namely, that analytic continuation can be applied, in the present case, so as to obtain useful expressions in the range of energies where the infinite series diverges.

This graph-based method can be further generalized for a complex, nonlinear structure of bridge. First, we note that each column of dots in Fig. 7 can be "condensed" into a larger vertex, the graph can be simplified to a row of large vertices with nearest neighbor vertices connected by a "multiple line." In this way, any one path drawn on this new graph represents all the possible paths on the old graph passing the same bridge sites in the same order with any choices of molecular orbitals. It corresponds, thereby, to a matrix, and each additional step on this new graph involves multiplication of matrices. Now a complicated bridge is represented by a simpler graph: a dot is used for each bridge site (which has several molecular orbitals), and lines exist between sites that interact with each other. The way to obtain the corresponding terms of Green's function from paths is to multiply all the matrices of lines and dots in the order given by the paths, as was done for the scalar terms for paths on the original graphs described in Section C of the Appendix. This method should provide a different way of calculating an electronic coupling between donor and acceptor in protein or other complicated systems.

With both properties discussed above, this graphical method is potentially useful for a variety of applications.

\footnotetext{
${ }^{1}$ (a) For example, for ET in biological processes there is a review article by B. E. Bowler, A. L. Raphael, and H. B. Gray, Prog. Inorg. Chem. 38, 259 (1990) and references cited therein; C. C. Moser, J. M. Keske, K. Warncke, R. S. Farid, and P. L. Dutton, Nature 355, 796 (1992); I. Bertini, H. B. Gray, S. J. Lippard, and J. Valentine, Bioinorganic Chemistry (Uni-
} 
versity Science, Mill Valley, 1994); (b) for organic bridges, see, for example, G. L. Closs and J. R. Miller, Science 240, 440 (1988); (c) for theoretical works on the electronic coupling of long range ET, see, for example, S. Larsson and M. Braga, Int. J. Quantum Biol. Symp. S20, 65 (1993) and references cited therein; J. Logan and M. D. Newton, J. Phys. Chem. 92, 3049 (1988); M. D. Newton, Chem. Rev. 91, 767 (1991), and references cited therein; J. R. Reimers and N. S. Hush, Chem. Phys. 146, 89 (1990); J. N. Onuchic, P. C. P. de Andrade, and D. N. Beratan, J. Chem. Phys. 95, 1131 (1991); P. Siddarth and R. A. Marcus, J. Phys. Chem. 97, 13078 (1993), and references cited therein; (d) P. Siddarth and R. A. Marcus, ibid. 96, 3213 (1992); 97, 2400 (1993).

${ }^{2}$ V. Mujica, M. Kemp, and M. A. Ratner, J. Chem. Phys. 101, 6849 (1994).

${ }^{3}$ J. W. Evenson and M. Karplus J. Chem. Phys. 96, 5272 (1992).

${ }^{4}$ D. N. Beratan and J. J. Hopfield J. Am. Chem. Sec. 1061584 (1984); D. N. Beratan, J. N. Onuchic, and J. J. Hopfield, J. Chem. Phys. 86, 4488 (1987).

${ }^{5}$ For example, see A. A. Stuchebrukhov, Chem. Phys. Lett. 225, 55 (1994); A. A. Stuchebrukhov and R. A. Marcus, J. Phys. Chem. 99, 7581 (1995).

${ }^{6}$ Experimental investigations of electron transfer across self-assembled monolayers on electrode surfaces include those of J. N. Richardson, S.R. Peck, L. S. Curtin, L. M. Tender, R. H. Terrill, M. T. Carter, R. W. Murray, G. K. Rowe, and S. E. Creager, J. Phys. Chem. 99, 766 (1995); R. J. Forster and L. R. Faulkner, J. Am. Chem. Soc. 116, 5444 (1994); 116, 9411 (1994); J. F. Smalley, S. W. Feldberg, C. E. D. Chidsey, M. R. Linford, M. D. Newton, and Y. P. Liu, J. Phys. Chem. 99, 13141 (1995); M. S. Ravenscroft, and H. O. Finklea, J. Phys. Chem. 98, 3843 (1994); Z. Q. Feng, S. Imabayashi, T. Kakiuchi, and K. Niki, J. Elec. Chem. 394, 149 (1995).

${ }^{7}$ C. E. D. Chidsey, Science 251, 919 (1991); 252, 631 (1991).

${ }^{8}$ A. M. Becka and C. J. Miller, J. Phys. Chem. 96, 2657 (1992).

${ }^{9}$ M. T. Carter, G. K. Rowe, J. N. Richardson L. M. Tender, R. H. Terrill, and R. W. Murray, J. Am. Chem. Soc. 117, 2896 (1995).

${ }^{10}$ V. Mujica, M. Kemp, and M. A. Ratner, J. Chem. Phys. 101, 6856 (1994); M. Kemp, V. Mujica, and M. A. Ratner, ibid. 101, 5172 (1994); M. Kemp, A. Roitberg, V. Mujica, T. Wanta, and M. A. Ratner, J. Phys. Chem. 100, 8349 (1996).

${ }^{11}$ (a) For studies involving large scale Green's function calculations, see, for example, S. S. Skourtis, J. J. Regan, and J. N. Onuchic, J. Phys. Chem. 98, 3379 (1994); S. S. Skourtis and J. N. Onuchic, Chem. Phys. Lett. 209, 171 (1993); 211, 282 (1993); A. A. S. da Gama, J. Theor. Biol. 142, 251 (1990); M. A. Ratner, J. Phys. Chem. 94, 4877 (1990); J. Malinsky and Y. Magarshak, ibid. 92, 2849 (1992); J. W. Evenson and M. Karplus, Science 262, 1247 (1993); (b) for other methods for long range ET, see A. Kuki and P. G. Wolynes, Science 236, 1647 (1987); L. S. Conrad, K. V. Mikklesen, M. K. Nielsen, and J. Ulstrup, Inorg. Chem. 29, 2808 (1990); S. F. Sneddon and C. L. Brooks, J. Am. Chem. Soc. 114, 8220 (1992); J. M. Gruschus and A. Kuki, J. Phys. Chem. 97, 5581 (1993); A. Okada, T. Kakitani, and J. Inoue, ibid. 99, 2946 (1995), among others.

${ }^{12}$ H. M. McConnell, J. Chem. Phys. 35, 508 (1961); the single band problem is also solved and discussed in detail by K. F. Herzfeld, ibid. 10, 508 (1942).

${ }^{13}$ S. Larsson, J. Am. Chem. Soc. 103, 4034 (1981); P. O. Löwdin, J. Mol. Spectrosc. 10,12 (1963); J. Math. Phys. 3, 969 (1962).

${ }^{14}$ E. N. Economou, Green's Functions in Quantum Physics, 2nd ed. (Springer, Berlin, 1990).

${ }^{15}$ S. G. Davison and M. Stęślicka, Basic Theory of Surface States (Clarendon, Oxford, 1992).

${ }^{16}$ The overlap integrals $S_{i j}$ of the atomic orbitals in tight-binding model were treated in the following way: (1) The overlap integrals were only nonzero within each $\mathrm{CH}_{2}$ bridge unit. (2) Within each $\mathrm{CH}_{2}$, the molecular orbitals are obtained by solving the linear equation $\mathbf{h p}=\mathbf{e} S \mathbf{p}$ for $\mathbf{e}$ and $\mathbf{p}$, where $\mathbf{h}$ is the Hamiltonian, $S$ is the overlap integral, $\mathbf{e}$ is a diagonal matrix with the eigenvalues as its diagonal matrix elements, and $\mathbf{p}$ is composed of column eigenvectors $\left(\mathbf{p}=\left(\vec{v}_{1}, \ldots, \vec{v}_{m}\right)\right)$. Solving the above equation is equivalent to solving the usual eigenvalue and eigenvector problem $\mathbf{h}^{\prime} \mathbf{p}^{\prime}=\mathbf{e p}^{\prime}$, where $\mathbf{h}^{\prime}=S^{-1 / 2} \mathbf{h} S^{-1 / 2}$ and $\mathbf{p}^{\prime}=S^{1 / 2} \mathbf{p} S^{1 / 2}$ $=S^{1 / 2}\left(\vec{v}_{1}, \ldots, \vec{v}_{m}\right) S^{1 / 2}$, and $\mathbf{p}^{\prime}$ forms an orthonormal basis set with which Eq. (8) is written.
${ }^{17}$ The first non-zero contribution of $G_{(1, n)}^{(n)}$ in the perturbative expansion of Eq. (12) is the first-order term $\mathscr{G}_{0}^{(n)} \mathscr{H}_{1}^{(n)} \mathscr{G}_{0}^{(n)}$. With Eqs. (13) and (18) the first-order contribution to $G_{(1, n)}^{(n)}$ can be obtained as $G_{(1, n-1)}^{(n-1)} \mathbf{v} \Delta^{-1}$. So $G_{(1, n)}^{(n)} \approx G_{(1,2)}^{(2)}\left(\mathbf{v} \Delta^{-1}\right)^{n-2}$. This expression is similar to Eq. (3), which is McConnell's estimation.

${ }^{18}$ If we denote $G_{(1, n)}^{(n)}=G_{(1,2)}^{(2)} \mathbf{x}_{n}$ with $\mathbf{x}_{n} \equiv \prod_{i=3}^{n} \mathbf{v} \Delta^{-1} N_{i}$, then $\mathbf{x}_{n}$ has solution $\mathbf{x}_{n}=\left[\alpha_{1}^{n-3} \mathbf{m}_{1}-\alpha_{2}^{n-3} \mathbf{m}_{2}\right]^{-1}$, for $n \geqslant 3$ with $\alpha_{1}=\Delta \mathbf{v}^{-1} / 2+\delta, \alpha_{2}$ $=\Delta \mathbf{v}^{-1} / 2-\delta, \mathbf{m}_{1}=\alpha_{1} \delta^{-1} \alpha_{1} / 2-\delta^{-1} \alpha_{1} \mathbf{v}^{T} \Delta^{-1} N_{2} / 2, \mathbf{m}_{2}=\alpha_{2} \delta^{-1} \alpha_{2} / 2$ - $\delta^{-1} \alpha_{2} \mathbf{v}^{T} \Delta^{-1} N_{2} / 2$, and $\delta$ is a matrix that satisfies $\delta^{2}=-\mathbf{v}^{T} \mathbf{v}^{-1}$ $+\left(\Delta \mathbf{v}^{-1}\right)^{2} / 4$. It is seen that upon matrix inversion to obtain $\mathbf{x}_{n}$, the eigenvalue that is the smallest in modulus of $\left[\alpha_{1}^{n-3} \mathbf{m}_{1}-\alpha_{2}^{n-3} \mathbf{m}_{2}\right]$ is the most important, while in numerical calculation of $\alpha_{1,2}^{n-3}$ for large $n$, the smaller eigenvalues of $\alpha_{1,2}$ are usually not preserved. In the present form, a specially designed algorithm would be needed in any numerical calculation using above expressions.

${ }^{19}$ The factor $\beta$ is conventionally defined as either of the following: $k_{\mathrm{ET}}$ $\propto e^{-\beta d}$, or $|\bar{V}|^{2} \propto e^{-\beta d}$ (Eq. (30)). They are slightly different because the reorganizational energy $\lambda$ is dependent of distance. Here, the latter definition was used, with $d$ being taken as the number of methylene units.

${ }^{20}$ For integrating over the electronic energy levels in the metal the weighting function in Eq. (6) of R.A. Marcus, J. Chem. Phys. 43, 679 (1965) is used, noting that only the second two factors there vary much with the $\epsilon$ in Eq. (6) there.

${ }^{21}$ R. A. Marcus, J. Chem. Soc. Faraday Trans. 92, 3905 (1996).

${ }^{22}$ R. A. Marcus and N. Sutin, Biochim. Biophys. Acta 811, 265 (1985).

${ }^{23}$ For treating the electronic matrix elements a method similar to that in $\mathrm{H}$. Ou-Yang, B. Källebring, and R. A. Marcus J. Chem. Phys. 98, 7565 (1993) is used.

${ }^{24}$ R. Hoffmann, J. Chem. Phys. 39, 1397 (1963).

${ }^{25}$ J. Hautman and M. L. Klein, J. Chem. Phys. 91, 4994 (1989).

${ }^{26}$ N. Camillone III, C. E. D. Chidsey, G.-Y. Liu, and G. Scoles, J. Chem. Phys. 98, 3503 (1993).

${ }^{27}$ International Union of Crystallography, Struct. Rep. 20, 550 (1956); 35B, 550 (1970).

${ }^{28}$ See AIP Document No. PAPS JCPSA-106-584-19 for 19 pages of a list of the coordinate used for the calculation and a figure showing the geometry of the principal chain and a neighboring chain. Order by PAPS number and journal reference from American Institute of Physics, Physics Auxiliary Publication Service, Carolyn Gehlbach, 500 Sunnyside Boulevard, Woodbury, NY 11797-2999. Fax: 516-576-2223, e-mail paps@aip.org. The price is $\$ 1.50$ for each microfiche (98 pages) or $\$ 5.00$ for photocopies of up to 30 pages, and $\$ 0.15$ for each additional page over 30 pages. Airmail additional. Make checks payable to the American Institute of Physics.

${ }^{29}$ R. Parsons, Pure Appl. Chem. 37, 501 (1974).

${ }^{30} \mathrm{~S}$. Trasatti, Electrochimica Acta 35, 269 (1990).

${ }^{31}$ N. W. Ashcroft and N. D. Mermin, Solid State Physics (Saunders, New York, 1976), p. 354

${ }^{32}$ S. Trasatti, Pure Appl. Chem 58, 955 (1986).

${ }^{33}$ CRC Handbook of Chemistry and Physics, 73rd ed., edited by D. R. Lide (Chemical Rubber, Cleveland, 1992-93).

${ }^{34}$ K. Seki, N. Ueno, U. O. Karlsson, R. Engelhardt, and E. E. Koch, Chem. Phys. 105, 247 (1986); Ch. Zubrägel, F. Schneider, M. Neumann, G. Hähner, Ch. Wöll, and M. Grunze, Chem. Phys. Lett. 219, 127 (1994).

${ }^{35}$ K. J. Less and E. G. Wilson, J. Phys. C 6, 3110 (1973); M. Fujihira and H. Inokuchi, Chem. Phys. Lett. 17, 554 (1972).

${ }^{36}$ The calculation of effective coupling $\overline{\left|H_{D k}\right|^{2}}$ does not depend on $\lambda$ (Eqs. (43) and (44)), so the calculation for various of chain lengths is a straightforward generalization without considering the dependence of $\lambda$ on the chain length $n$. For calculating the reaction rate with Eq. (30), proper values of $\lambda$ are needed for different chain lengths, which has been discussed in the following reference: Y. P. Liu and M. D. Newton, J. Phys. Chem. 98, 7162 (1994).

${ }^{37}$ A. P. Cracknell and K. C. Wong, The Fermi Surface, Its Concept, Determination and Use in the Physics of Metals (Clarendon, Oxford, 1973).

${ }^{38}$ C. Goldman, Phys. Rev. A 43, 4500 (1991). 